QUARTERLY OF APPLIED MATHEMATICS

VOLUME LXIV, NUMBER 4

DECEMBER 2006, PAGES 749-795

S $0033-569 \mathrm{X}(06) 01036-\mathrm{X}$

Article electronically published on September 14, 2006

\title{
ELECTROMAGNETIC INVERSE PROBLEMS INVOLVING DISTRIBUTIONS OF DIELECTRIC MECHANISMS AND PARAMETERS
}

BY

H. T. BANKS (Center for Research in Scientific Computation, North Carolina State University, Raleigh, North Carolina 27695-8205)

AND

N. L. GIBSON (Center for Research in Scientific Computation, North Carolina State University, Raleigh, North Carolina 27695-8205)

Abstract. We consider electromagnetic interrogation problems for complex materials involving distributions of polarization mechanisms and also distributions for the parameters in these mechanisms. A theoretical and computational framework for such problems is given. Computational results for specific problems with multiple Debye mechanisms are given in the case of discrete, uniform, log-normal, and log-bi-Gaussian distributions.

1. Introduction. For at least the past century [52, 53, scientific investigators have sought to understand what happens to electromagnetic fields (and how to mathematically model the associated phenomena) when they are introduced into complex materials such as biotissue and more general dielectrics, conductors and magnetics. More specifically, a fundamental question is how to model dispersion and dissipation of the fields in these complex materials. This has most often led to the use of Maxwell's equations in a nonvacuum environment which entails constitutive relationships for polarization (in dielectrics), magnetization (in magnetic materials) and conductivity. We focus here on modeling polarization in dielectric materials for which we develop a new modeling framework. Even though we treat only polarization as our dispersive mechanism in our formulation (adopting Ohm's law for conductivity and considering nonmagnetic materials), the approach is sufficiently general so as to be readily extended to treat magnetization and conductivity in materials (each in some type of convolution representation involving susceptibility kernels, e.g., see 2, 3]). We develop a framework that allows not only uncertainty (through

Received April 13, 2006.

2000 Mathematics Subject Classification. Primary 35Q60, 93A30, 49 N45.

Key words and phrases. Electromagnetic interrogation, pulsed antenna source microwaves, inverse problems, complex dielectric materials, distributions of relaxation parameters and mechanisms.

E-mail address: htbanks@ncsu.edu

E-mail address: ngibson@ncsu.edu 
distributions of parameters representing molecular variability) at the molecular level, but also allows for the presence of multiple polarization mechanisms in the material.

We first explain a conceptual framework in the context of the 3-D Maxwell equations in a dielectric material. In particular, we use Maxwell's equations which govern the electric field $\mathbf{E}$ and the magnetic field $\mathbf{H}$ in a domain $\mathcal{D}=\Omega_{0} \cup \Omega$ with charge density $\rho$ in the material $\Omega$ while the ambient $\Omega_{0}$ is treated as a vacuum. Thus we first consider the system

$$
\left\{\begin{array}{l}
\text { (i) } \frac{\partial \mathbf{D}}{\partial t}+\mathbf{J}-\nabla \times \mathbf{H}=0, \text { in }(0, T) \times \mathcal{D}, \\
\text { (ii) } \frac{\partial \mathbf{B}}{\partial t}+\nabla \times \mathbf{E}=0, \text { in }(0, T) \times \mathcal{D} \\
\text { (iii) } \nabla \cdot \mathbf{D}=\rho, \text { in }(0, T) \times \mathcal{D} \\
\text { (iv) } \nabla \cdot \mathbf{B}=0, \text { in }(0, T) \times \mathcal{D} \\
\text { (v) } \quad \mathbf{E} \times \mathbf{n}=0, \text { on }(0, T) \times \partial \mathcal{D}, \\
\text { (vi) } \quad \mathbf{E}(0, \mathbf{x})=0, \quad \mathbf{H}(0, \mathbf{x})=0, \text { in } \mathcal{D}
\end{array}\right.
$$

As usual, the current $\mathbf{J}$ is composed of the source current $\mathbf{J}_{s}$ and the conductive current $\mathbf{J}_{c}$. Within the domain we have constitutive relations that relate the flux densities $\mathbf{D}, \mathbf{B}$ and the conductive current $\mathbf{J}_{c}$ to the electric and magnetic fields. We have

$$
\left(\begin{array}{ll}
\text { (i) } & \mathbf{D}=\epsilon_{0} \mathbf{E}+\mathbf{P}_{\mathbf{T}} I_{\Omega}, \\
\text { (ii) } & \mathbf{B}=\mu_{0} \mathbf{H}, \\
\text { (iii) } & \mathbf{J}_{c}=\sigma \mathbf{E} I_{\Omega} .
\end{array}\right.
$$

In (2), $I_{\Omega}$ denotes the indicator function on the dielectric medium $\Omega$. Thus $\mathbf{J}_{c}=0$ in the ambient or air. The total electric polarization $\mathbf{P}_{\mathbf{T}}$ is given by

$$
\mathbf{P}_{\mathbf{T}}=\mathbf{P}_{\mathrm{I}}+\mathbf{P}=\epsilon_{0} \chi \mathbf{E}+\mathbf{P},
$$

where $\mathbf{P}_{\mathrm{I}}$ is the instantaneous polarization due to the interface between $\Omega_{0}$ and $\Omega$ and $\mathbf{P}$ is the material or dielectric polarization. Hence the constitutive law (2, i) in $\Omega$ becomes

$$
\mathbf{D}=\epsilon_{0} \epsilon_{r} \mathbf{E}+\mathbf{P}
$$

where $\epsilon_{r}=(1+\chi)$ is the relative permittivity of the dielectric medium.

Our main focus in this presentation is the dielectric polarization $\mathbf{P}$, which we assume has the general convolution form

$$
\mathbf{P}(t, \mathbf{x})=g \star \mathbf{E}(t, \mathbf{x})=\int_{0}^{t} g(t-s, \mathbf{x}) \mathbf{E}(s, \mathbf{x}) d s,
$$

where $g$ is the general dielectric response function (DRF). In every practical example (Debye, Lorentz, etc.) DRFs are parameter dependent as well as time (and possibly space) dependent; we represent this as $g=g(t, \mathbf{x} ; \nu)$, where typically $\nu=\left(\epsilon_{\infty}, \epsilon_{s}, \tau\right)$ contains parameters such as the high frequency limit dielectric permittivity $\epsilon_{\infty}$, the static permittivity $\epsilon_{s}$, and the relaxation time $\tau$. Examples of often-used DRFs are the Debye [11, 24, 30] in a material region $\Omega$ defined in the time domain by

$$
g(t, \mathbf{x})=\epsilon_{0}\left(\epsilon_{s}-\epsilon_{\infty}\right) / \tau e^{-t / \tau},
$$


the Lorentz [11, 24, 41] given by

$$
g(t, \mathbf{x})=\left(\epsilon_{0} \omega_{p}^{2} / \nu_{0}\right) e^{-t / 2 \tau} \sin \left(\nu_{0} t\right)
$$

and the Cole-Cole [24, 28, 33, 39, 47] defined by

$$
g(t, \mathbf{x})=\mathcal{L}^{-1}\left\{\frac{\epsilon_{0}\left(\epsilon_{s}-\epsilon_{\infty}\right)}{1+(s \tau)^{\alpha}}\right\}=\frac{1}{2 \pi i} \int_{\zeta-i \infty}^{\zeta+i \infty} \frac{\epsilon_{0}\left(\epsilon_{s}-\epsilon_{\infty}\right)}{1+(s \tau)^{\alpha}} e^{s t} d s
$$

where $\mathcal{L}$ is the Laplace transform.

These DRFs also play a fundamental role in convolution representations such as (3) for nonlinear polarization laws [8, 20, 21, 25, 41] of Kerr type and Raman scattering [41]. While the ideas we describe here on distributions of relaxation times and mechanisms can readily be used to treat such nonlinear polarization laws, we shall in this paper concentrate on linear constitutive laws involving multiple relaxation parameters and multiple dispersive mechanisms in materials in the presence of multiple interrogating frequencies.

The macroscopic polarization model (3) can be derived from the microscopic dipole, electron cloud, etc., formulations arrived at by passing to a limit over the molecular population [31. However, such derivations tacitly assume that one has similar individual (molecular, dipole, etc.) parameters; that is, all dipoles, molecules, "electron clouds", etc. have the same relaxation parameters, plasma frequencies, etc. Historically, such models based on molecular level homogeneity throughout the material have often not performed well when trying to compare models with experimental data. Indeed, in 1907 Von Schweidler [24, 52] observed the need to assume multiple relaxation times when considering experimental data and in 1913 Wagner [24, 53, proposed continuous distributions of relaxation times. This idea was subsequently visited by Williams and Ferry [57] in comparing the behavior of viscoelastic polymers and dielectric materials. In the past half century intensive experimental efforts [32, 33, 34, 35, 36, 39, 46] have been pursued in describing data for complex materials with distributions of dielectric parameters (especially relaxation times in multiple Debye [32] or multiple Lorentz [41] mechanisms) in the frequency domain. A significant amount of this work is reviewed in the survey paper by Foster and Schwan 32. There are now incontrovertible experimentally based arguments for distributions of relaxation parameters in mechanisms for heterogeneous materials. Moreover, there is compelling evidence of the presence of multiple mechanisms in complex materials such as tissue and modern polymeric composites. These multiple mechanisms may involve interfacial polarization, dipolar orientation, ionic diffusion (e.g., see pp. 40, 49, 57 of [32]) and may often require a selection of several types of distributional representations from examples such as the fractional power laws of the Cole-Cole [26, 27, 32, 36, 47, the log normal, the uniform, as well as the Debye and Lorentz (although the fractional power law of Cole-Cole is more the rule rather than the exception - p. 39, 32]). These multiple mechanisms are likely present in some weighted combination (e.g., see [37. and p. 369, 41]) and often are manifested in a frequency-dependent manner. It is therefore advantageous to consider interrogation or inverse problems with multiple frequencies (e.g., ranging from $\mathrm{RF}\left(10^{6}\right)$ to $\mathrm{GHz}\left(10^{10}\right)$ ) or broadband excitation signals. 
To allow for a distribution $F$ of parameters $\nu$ over some admissible set $\mathcal{N}$, we generalize the polarization law (3) to

$$
\mathbf{P}(t, \mathbf{x} ; F)=h \star \mathbf{E}(t, \mathbf{x})=\int_{0}^{t} \int_{\mathcal{N}} g(t-s, \mathbf{x} ; \nu) \mathbf{E}(s, \mathbf{x}) d F(\nu) d s .
$$

We expect to chose $F$ from (or from a subspace of) the space $\mathcal{F}=\mathfrak{P}(\mathcal{N})$ of all probability measures $F$ on $\mathcal{N}$.

We further generalize (4) to allow for dielectric materials with multiple mechanisms or multiple DRFs (i.e., heterogeneous molecular structures) by considering a family $\mathcal{G}$ of possible DRFs and distributions $M$ over this family. This leads to the polarization constitutive relationship

$$
\begin{aligned}
\mathbf{P}(t, \mathbf{x} ; M, F) & =\int_{0}^{t} \int_{\mathcal{G}} \int_{\mathcal{N}} g(t-s, \mathbf{x} ; \nu) \mathbf{E}(s, \mathbf{x}) d F(\nu) d M(g) d s \\
& =\int_{0}^{t} K(t-s, \mathbf{x} ; M, F) \mathbf{E}(s, \mathbf{x}) d s,
\end{aligned}
$$

where for $F \in \mathcal{F}=\mathfrak{P}(\mathcal{N})$ and $M \in \mathcal{M}=\mathfrak{P}(\mathcal{G}), K$ is defined by

$$
K(t-s, \mathbf{x} ; M, F)=\int_{\mathcal{G}} \int_{\mathcal{N}} g(t-s, \mathbf{x} ; \nu) d F(\nu) d M(g) .
$$

When we use (5) and (6) in the Maxwell system (11)-(2), we are led to a system of partial differential equations where lower-order terms (in time) depend on probability measures. These measures are now the "parameters" that characterize the material dielectric properties which one must estimate or identify in interrogation problems.

With the recently growing interest in incorporating uncertainty into models and systems, the need to employ dynamics with probabilistic structures has received increased emphasis. In particular, systems with probability measures embedded in the dynamics (problems involving aggregate dynamics as discussed in [8]) have become important in applications in biology [9, 10, 8], electromagnetics [12] and hysteretic [16, 17, 42, 43] and polymeric [18, 19, 22] materials. These systems (in the case of first-order ordinary differential equations) have the form

$$
\dot{x}(t)=f(t, x(t), F),
$$

where $F$ is a probability distribution or measure. In fact such systems are not new and arise in relaxed or chattering control problems [44, 45, 49, 54, 55, 56] wherein the controls are probability measures. Indeed, such systems date back to the seminal work of L.C. Young on generalized curves in the calculus of variations [58, 59.

In the next section we formulate an inverse problem for the Maxwell system with the general polarization law (5) and discuss theoretical and computational aspects of this problem. We then report on our initial computational efforts on a 1-dimensional version of the problem of finding the underlying polarization in a slab of material using signals reflected from a front air/material interface and a metal backing. We present numerical results for inverse problems involving Maxwell's equations with absorbing left boundary condition, a superconducting right boundary condition and a general polarization term which includes uncertainty in the dielectric parameters. We attempt to determine an 
unknown distribution of parameters which describes the dielectric properties of the material. We explore both discrete and continuous distributions; for the continuous case appropriate parameterizations and discretizations are used.

2. A general inverse problem. We consider the Maxwell system (1)-(2) with polarization $\mathbf{P}=\mathbf{P}(t, \mathbf{x} ; M, F)$ given by (15). Let

$$
\mathbf{z}(t, \mathbf{x} ; M, F)=\left(\begin{array}{c}
\mathbf{E}(t, \mathbf{x} ; M, F) \\
\mathbf{H}(t, \mathbf{x} ; M, F)
\end{array}\right)
$$

with $(t, \mathbf{x}) \rightarrow \mathbf{z}(t, \mathbf{x} ; M, F)$ mapping from $(0, T) \times \Omega$ to $R^{6}$. We assume we are given data $\overline{\mathbf{d}}=\left\{\mathbf{d}_{i}\right\}_{i=1}^{n}$ corresponding to observations of $\mathcal{C}_{A} \mathbf{z}\left(t_{i}, \cdot ; M, F\right)$. Here $\mathcal{C}_{A}$ denotes evaluation of one or more components of $\mathbf{E}$ or $\mathbf{H}$ at an antenna $\left\{\mathbf{x}_{A}\right\}$. We use this data to estimate the distributions $M$ and $F$ in an ordinary least squares (OLS) formulation, seeking to minimize

$$
J(M, F)=\sum_{i=1}^{n}\left|\mathcal{C}_{A} \mathbf{z}\left(t_{i}, \cdot ; M, F\right)-\mathbf{d}_{i}\right|^{2}
$$

over $(M, F) \in \mathcal{M} \times \mathcal{F}=\mathfrak{P}(\mathcal{G}) \times \mathfrak{P}(\mathcal{N})$. We thus seek to find $(\hat{M}, \hat{F})$ such that

$$
(\hat{M}, \hat{F})=\arg \min \{J(M, F): M \in \mathcal{M}, F \in \mathcal{F}\} .
$$

We note that while for simplicity we use an OLS formulation here, most of the results discussed below could readily be developed in the context of other standard estimation formulations such as maximum likelihood estimators (MLE), weighted least squares (WLS), or generalized least squares (GLS).

For theoretical and computational purposes, one needs a topology on $\mathcal{M}$ and $\mathcal{F}$ and for this we choose the Prohorov metric $\rho_{*}$ of weak ${ }^{*}$ convergence in $\mathcal{M}$ and $\mathcal{F}$ when they are considered as subsets of the topological duals $C_{B}^{*}(\mathcal{G})$ and $C_{B}^{*}(\mathcal{N})$ of the spaces $C_{B}(\mathcal{G})$ and $C_{B}(\mathcal{N})$ of bounded continuous functions on $\mathcal{G}$ and $\mathcal{N}$, respectively [5, 8 . That is, $F_{k} \rightarrow F$ in the $\rho_{*}$ metric if and only if

$$
\int_{\mathcal{N}} \phi(\nu) d F_{k}(\nu) \rightarrow \int_{\mathcal{N}} \phi(\nu) d F(\nu)
$$

for all $\phi \in C_{B}(\mathcal{N})$, i.e., all bounded continuous $\phi$ on $\mathcal{N}$; a similar result holds for $M_{k} \rightarrow M$. It is known [5, 8] that if $\mathcal{G}$ and $\mathcal{N}$ are complete metric spaces, then $\mathcal{M}=\mathfrak{P}(\mathcal{G})$ and $\mathcal{F}=\mathfrak{P}(\mathcal{N})$ taken with the Prohorov metric $\rho_{*}$ are complete metric spaces. Moreover, if $\mathcal{G}$ and/or $\mathcal{N}$ are compact, then so are $\mathcal{M}$ and/or $\mathcal{F}$. Using these properties and arguments similar to those in [5, 15], the following problem stability results can be proven.

Theorem 1. Suppose $(M, F) \rightarrow \mathcal{C}_{A} \mathbf{z}(t, \cdot ; M, F)$ is continuous on $\mathcal{M} \times \mathcal{F}$ and suppose that $\mathcal{G}$ and $\mathcal{N}$ are compact. Then solutions $(\hat{M}, \hat{F})$ of minimizing (7) exist (generally, nonuniquely) and are continuous in the data $\overline{\mathbf{d}}$ in the following sense. Suppose $\left(M^{*}(\overline{\mathbf{d}}), F^{*}(\overline{\mathbf{d}})\right)$ and $\left(M^{*}\left(\overline{\mathbf{d}}^{k}\right), F^{*}\left(\overline{\mathbf{d}}^{k}\right)\right)$ are the solution sets of minimizing (7) for data $\overline{\mathbf{d}}$ and $\overline{\mathbf{d}}^{k}$, respectively, where $\overline{\mathbf{d}}^{k} \rightarrow \overline{\mathbf{d}}$ as $k \rightarrow \infty$. Then

$$
\operatorname{dist}\left[\left(M^{*}\left(\overline{\mathbf{d}}^{k}\right), F^{*}\left(\overline{\mathbf{d}}^{k}\right)\right),\left(M^{*}(\overline{\mathbf{d}}), F^{*}(\overline{\mathbf{d}})\right)\right] \rightarrow 0
$$

as $k \rightarrow \infty$, where $\operatorname{dist}[\cdot, \cdot]$ is the Hausdorff distance in the metric space $\mathcal{M} \times \mathcal{F}$. 
The problem of minimizing (77) over $\mathcal{M} \times \mathcal{F}$ is in general an infinite-dimensional optimization problem that poses formidable computational challenges. But we note that the set $\mathcal{G}$ of possible DRFs can in almost all cases be taken as a fixed finite set, i.e., $\mathcal{G}=\left\{g_{1}, g_{2}, \ldots, g_{K}\right\}$, which means that $\mathcal{M}$ would be given by

$$
\mathcal{M}=\left\{M(g) \in \mathfrak{P}(\mathcal{G}) \mid d M(g)=\sum_{j=1}^{K} a_{j} d \Delta_{g_{j}}(g), a_{j} \geq 0, \sum_{j} a_{j}=1\right\},
$$

where $\Delta_{g_{j}}$ is the Dirac measure with a single atom at $g_{j}$, i.e., $d \Delta_{g_{j}}(g)=\delta_{g_{j}}(g) d g$. Thus, this part of the optimization reduces to one over a closed bounded convex set in Euclidean space. The minimization over $\mathcal{F}=\mathfrak{P}(\mathcal{N})$ is more interesting since in general one expects $\mathcal{N}$ to involve a continuum of (vector) parameters. We illustrate the possibilities with $\mathcal{N}=\mathcal{T}=\left\{\tau \mid \tau \in\left[\tau_{a}, \tau_{b}\right]\right\}$ for $\tau$ the relaxation parameter in, for example, a Debye or Lorentz mechanism. There are a number of ways this problem could be approached:

(1) assume that $F$ is discrete, having the fixed form $d F(\tau)=\sum \alpha_{j} \delta_{\tau_{j}}(\tau) d \tau$ and seek to find $\left(\alpha_{j}, \tau_{j}\right)$ minimizing (77) where $\alpha_{j} \geq 0, \sum \alpha_{j}=1, \tau_{j} \in\left[\tau_{a}, \tau_{b}\right]$;

(2) assume that $F$ is (absolutely) continuous and given in parametric form $d F(\tau)=f(\tau, \mu, \sigma) d \tau$ where $f$ is known (e.g., normal, log-normal, uniform, etc.) and seek to find $(\mu, \sigma)$;

(3) assume $F$ does not have a specific parametric form and seek to find the general form for $F$ through the optimization of (7).

In the first two cases above, one effectively reduces the inverse problem to a computationally tractable (one hopes!-we explore these ideas computationally in subsequent discussions below) optimization problem that is finite dimensional. The third case remains infinite dimensional in nature and one must develop approximation ideas that lead to implementable computational algorithms. In [5], the authors developed approximation ideas based on density results for measures arising in probability theory. We only outline those here, referring the reader to [5] for more details and proofs.

To develop approximation ideas for the nonparametric case, we first consider a family of partition points $\mathcal{T}^{N}=\left\{q_{j}^{N}\right\}_{j=1}^{N}, N=1,2, \ldots$, such that $\bigcup_{N=1}^{\infty} \mathcal{T}^{N}$ is dense in $\mathcal{T}$. Then define

$$
\begin{aligned}
\mathcal{F}^{N} & =\mathfrak{P}^{N}(\mathcal{T}) \\
& =\left\{F^{N} \in \mathfrak{P}(\mathcal{T}) \mid d F^{N}(\tau)=\sum_{j=1}^{N} p_{j}^{N} \delta_{q_{j}^{N}}(\tau) d \tau, q_{j}^{N} \in \mathcal{T}^{N}, p_{j}^{N} \text { rational, } \sum p_{j}^{N}=1\right\} .
\end{aligned}
$$

It can be argued that $\bigcup_{N=1}^{\infty} \mathcal{F}^{N}$ is dense in $\mathcal{F}$ in the Prohorov metric $\rho_{*}$. Moreover, if $\mathcal{T}$ is compact, one can prove a method stability theorem (see [5, 8) similar to Theorem 1 above. Specifically, let $F_{N}^{*}(\overline{\mathbf{d}})$ be the set of solutions obtained in minimizing (7) with $\mathcal{F}$ replaced by $\mathcal{F}^{N}$. Then the method stability theorem guarantees

$$
\operatorname{dist}\left[\left(M^{*}\left(\overline{\mathbf{d}}^{k}\right), F_{N}^{*}\left(\overline{\mathbf{d}}^{k}\right)\right),\left(M^{*}(\overline{\mathbf{d}}), F^{*}(\overline{\mathbf{d}})\right)\right] \rightarrow 0
$$

as $N, k \rightarrow \infty$. 
More generally, one may wish to consider only classes of distributions $\mathcal{F}$ that arise from density functions, i.e., absolutely continuous distributions in

$$
\mathcal{F}_{A C}=\left\{F \in \mathcal{F} \mid F^{\prime}=f, f \in \mathcal{F}_{\text {weak }}\right\},
$$

where $\mathcal{F}_{\text {weak }}$ is a given weakly compact subset of $L^{2}(\mathcal{T})$. For example, $\mathcal{F}_{\text {weak }}$ could be any given closed, convex, bounded subset of $L^{2}(\mathcal{T})$. It is proven in 22] that sets $\mathcal{F}_{A C}$ defined in this way are compact (in the $\rho_{*}$ metric) subsets of $\mathcal{F}$, and the corresponding existence and stability results of Theorem 1 hold for problems using $\mathcal{F}_{A C}$ in place of $\mathcal{F}$ in the optimization for (7). But once again these are infinite dimensional in nature and approximations are needed. We note that although the computational framework described above utilizing Dirac measures is also valid here, it is often desirable to develop "smoother" approximations to elements of $\mathcal{F}_{A C}$. In particular, suppose that $f \in \mathcal{F}_{\text {weak }}$ and $F \in \mathcal{F}=\mathfrak{P}(\mathcal{T})$ with $F=\int f$. Since $\mathcal{F}_{\text {weak }}$ is a subset of $L^{2}(\mathcal{T})$, we can use any number of types of splines to formulate an approximation to $f$, i.e., let $\left\{S_{j}^{N}\right\}_{j=1}^{N}, N=$ $1,2, \ldots$ be a standard spline family [15, 50, 51] (e.g., piecewise-linear, cubic, Hermite cubic, etc.) and

$$
f^{N}(\tau)=\sum_{j=1}^{N} w_{j}^{N} S_{j}^{N}(\tau)
$$

where the rational numbers $w_{j}^{N}$ are chosen so that $f^{N} \rightarrow f$ in $L^{2}(\mathcal{T})$. This implies that

$$
\int_{\mathcal{T}} \phi f^{N} d \tau \rightarrow \int_{\mathcal{T}} \phi f d \tau
$$

for all $\phi \in L^{2}(\mathcal{T})$, and hence for all $\phi \in C(\mathcal{T})$, which yields

$$
\rho\left(F^{N}, F\right) \rightarrow 0,
$$

where $F^{N}=\int f^{N}$. Thus defining

$$
\mathcal{F}_{S P L}^{N}=\left\{h \in L^{2}(\mathcal{T}) \mid h(\tau)=\sum_{j=1}^{N} w_{j}^{N} S_{j}^{N}(\tau)\right\},
$$

we can conclude that the set

$$
\tilde{\mathcal{F}}_{A C}=\left\{F \in \mathcal{F} \mid F=\int f, f \in \bigcup_{N=1}^{\infty} \mathcal{F}_{S P L}^{N}\right\}
$$

is dense in $\mathcal{F}_{A C}$ in the $\rho_{*}$-metric. Hence spline families provide an alternative way to approximate elements of $\mathcal{F}_{A C}$ in our computational work. We note that in this case the approximating elements are not probability distributions themselves. Once again one can state and prove a method stability theorem [15] using these spline approximations. That is:

TheOREm 2. Suppose $\mathcal{T}$ is compact and the solutions $\mathcal{C}_{A} \mathbf{z}(t, \cdot ; M, F)$ are continuous. Let $\mathcal{F}_{\text {weak }}, \mathcal{F}_{A C}$, and $\mathcal{F}_{S P L}^{N}$ be as defined above with $\left(M^{*}(\overline{\mathbf{d}}), F_{N}^{*}(\overline{\mathbf{d}})\right)$ the solution sets for minimizing (7) with $\mathcal{F}$ replaced by $\mathcal{F}_{S P L}^{N}$. Then for $\overline{\mathbf{d}}^{k} \rightarrow \overline{\mathbf{d}}$ we have

$$
\operatorname{dist}\left[\left(M^{*}\left(\overline{\mathbf{d}}^{k}\right), F_{N}^{*}\left(\overline{\mathbf{d}}^{k}\right)\right),\left(M^{*}(\overline{\mathbf{d}}), F^{*}(\overline{\mathbf{d}})\right)\right] \rightarrow 0
$$


as $N, k \rightarrow \infty$. Thus the solutions depend continuously on the data, and the approximate problems are method stable.

To illustrate the computational aspects of the ideas presented in this section, we turn next to a 1-D version of the inverse problem and present results in the next several sections on the use of discrete and continuous distributions in the polarization laws.

3. The 1-D problem formulation. For our initial numerical efforts, we turned to the 1-D example as explained in detail in [11. Under the assumptions detailed there, one obtains a domain as depicted in Figure 1

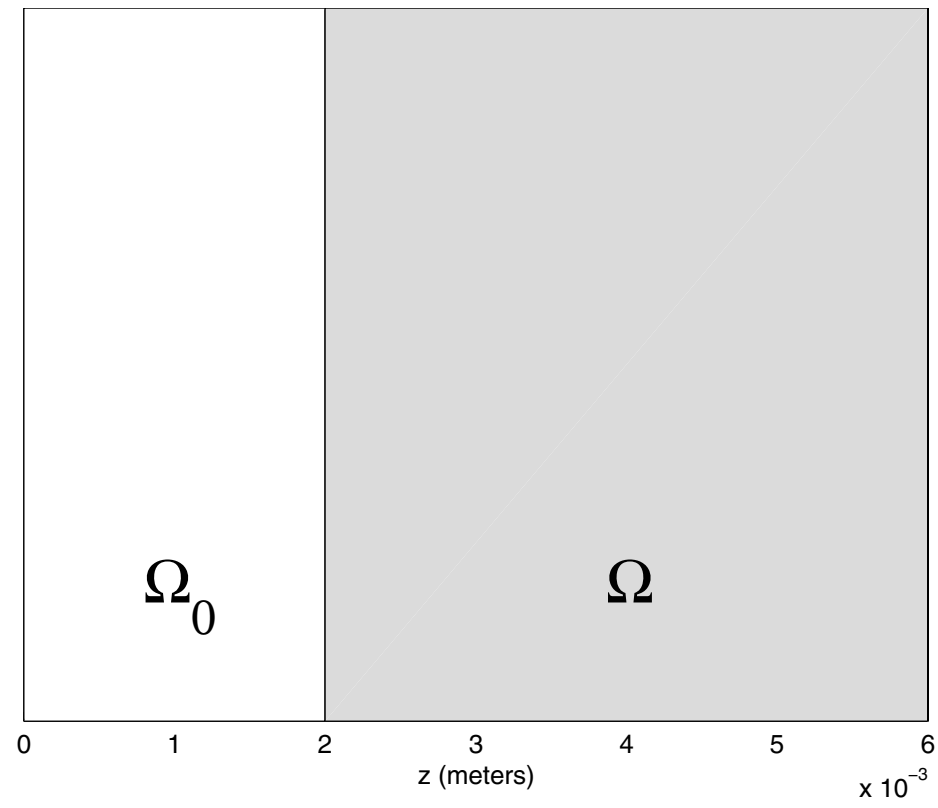

FIG. 1. The 1-D domain.

Restricting to one dimension, using $D=\epsilon E+P$, and adopting the notation $\dot{E}=\frac{\partial E}{\partial t}$, $E^{\prime}=\frac{\partial E}{\partial z}$, we can write Maxwell's equations in second-order form as

$$
\mu_{0} \epsilon \ddot{E}+\mu_{0} I_{\Omega} \ddot{P}+\mu_{0} \sigma I_{\Omega} \dot{E}-E^{\prime \prime}=-\mu_{0} \dot{J}_{s} \text { in } \Omega \cup \Omega_{0},
$$

where $E$ is the $\hat{k}$ or $z$ component of the electric field, $P$ is the media's macroscopic electric polarization, $J_{s}$ is the interrogating signal, $\Omega$ is the domain of the material under investigation, $\Omega_{0}$ is the ambient domain (considered a vacuum), and $\sigma=\sigma(z)$ is the conductivity of the material. Note we are considering a nonmagnetic material containing no charge distribution $(\rho=0)$. Also, let $\epsilon=\epsilon_{0}\left(1+I_{\Omega}\left(\epsilon_{r}-1\right)\right)$, where $\epsilon_{r}=\epsilon_{r}(z)$ is the dielectric permittivity. The values $\mu_{0}$ and $\epsilon_{0}$ are the magnetic permeability and the electric permittivity of free space, respectively. See [1] for more details. We employ 
the "method of mappings" [11, 48 to obtain a computational domain of $z=[0,1]$. The boundary conditions that we are assuming are absorbing at $z=0$ (to provide a finite computational window) and superconducting at $z=1$ (representing a metal backing):

$$
\begin{aligned}
{\left[\dot{E}-c E^{\prime}\right]_{z=0} } & =0, \\
E(t, 1) & =0 .
\end{aligned}
$$

We assume homogeneous initial conditions

$$
\begin{aligned}
& E(0, z)=0, \\
& \dot{E}(0, z)=0 .
\end{aligned}
$$

For implementation, we scale time by $\tilde{t}=c t$ and the polarization by $\tilde{P}=P / \epsilon_{0}$ for convenience. The actual dimensions of the domains considered in this report depend on the interrogating frequency $\omega$. In particular, for $\omega=2 \pi \times 10^{11}$ we consider a material slab of thickness $.004 \mathrm{~m}$ preceded by a vacuum of depth $.002 \mathrm{~m}$. We scale these dimensions as the wavelength scales when we change interrogating frequencies.

Converting the system (8) to weak form (and dropping the notation) we obtain

$$
\left\langle\epsilon_{r} \ddot{E}(t, \cdot), \phi\right\rangle+\left\langle I_{\Omega} \ddot{P}(t, \cdot), \phi\right\rangle+\left\langle\eta_{0} \sigma I_{\Omega} \dot{E}(t, \cdot), \phi\right\rangle-\left\langle E^{\prime \prime}(t, \cdot), \phi\right\rangle=-\left\langle\eta_{0} \dot{J}_{s}(t, \cdot), \phi\right\rangle,
$$

where $\eta_{0}=\sqrt{\mu_{0} / \epsilon_{0}}$ and $\phi \in V=H_{R}^{1}(0,1)=\left\{\phi \in H^{1}(0,1): \phi(1)=0\right\}$. Finally, we integrate by parts, and apply the boundary conditions (9) to get

$$
\begin{aligned}
\left\langle\epsilon_{r} \ddot{E}(t, \cdot), \phi\right\rangle+\left\langle I_{\Omega} \ddot{P}(t, \cdot), \phi\right\rangle+ & \left\langle\eta_{0} \sigma I_{\Omega} \dot{E}(t, \cdot), \phi\right\rangle+\left\langle E^{\prime}(t, \cdot), \phi^{\prime}\right\rangle+\dot{E}(t, 0) \phi(0) \\
& =-\left\langle\eta_{0} \dot{J}_{s}(t, \cdot), \phi\right\rangle .
\end{aligned}
$$

To describe the behavior of the electric polarization $P$, we may employ a general polarization kernel, or dielectric response function $g$, as follows:

$$
P(t, z)=\int_{0}^{t} g(t-s, z ; \tau) E(s, z) d s,
$$

where, for instance using a Debye polarization model,

$$
g(t ; \tau)=\epsilon_{0}\left(\epsilon_{s}-\epsilon_{\infty}\right) / \tau e^{-t / \tau}
$$

However, this presupposes that the material may be sufficiently defined by a single relaxation parameter $\tau$, which is generally not the case. In order to account for uncertainty in the polarization mechanisms, we allow for a distribution of the relaxation parameters. Thus, we define our polarization model in terms of a distribution dependent dielectric response function $h$,

$$
P(t, z)=\int_{0}^{t} h(t-s, z ; F) E(s, z) d s
$$

where $h$ is determined by a family of polarization laws, each described by a different parameter $\tau$, and therefore is given by

$$
h(t, z ; F)=\int_{\mathcal{T}} g(t, z ; \tau) d F(\tau)
$$

where $\mathcal{T}=\left[\tau_{1}, \tau_{2}\right] \subset(0, \infty)$. In particular, if the distribution $F$ were discrete, consisting of a single relaxation parameter, then we would again have (13). 
The macroscopic electric polarization becomes

$$
P(t, z)=\int_{0}^{t}\left[\int_{\mathcal{T}} g(t-s, z ; \tau) d F(\tau)\right] E(s, z) d s,
$$

or, interchanging integrals, we have

$$
P(t, z)=\int_{\mathcal{T}} \mathcal{P}(t, z ; \tau) d F(\tau),
$$

where

$$
\mathcal{P}(t, z ; \tau)=\int_{0}^{t} g(t-s, z ; \tau) E(s, z) d s
$$

is the polarization due to the relaxation parameter $\tau$. Thus assuming we have a computational method to compute (13), i.e., $\mathcal{P}$, we use this as a basis for approximating $P$, either directly in the discrete case, or using a quadrature rule in the continuous case.

The theory developed in Section 2 for general inverse problems can be directly applied to this 1-D problem once one has established continuity of the solution $E$ with respect to the measures $F$ in the space $\mathcal{F}=\mathfrak{P}(\mathcal{T})$ taken with the Prohorov metric. But these desired continuity results are given in [12].

3.1. Discrete distribution. Consider the discrete distribution given by

$$
d F(\tau)=\sum_{i=0}^{\ell} \frac{\delta\left(\tau_{i}\right)}{\ell+1} d \tau
$$

where $\delta$ represents the Dirac distribution, and $\tau_{i}=\tau_{a}+i \tau_{h}$ with $\tau_{h}=\left(\tau_{b}-\tau_{a}\right) / \ell$. Then we have

$$
P(t, z)=\sum_{i=0}^{\ell} \alpha_{i} \mathcal{P}\left(t, z ; \tau_{i}\right),
$$

where $\alpha_{i}=\frac{1}{\ell+1}$. Note that for this example, the nodes $\left(\tau_{i}\right)$ are linearly spaced and the weights or masses $\left(\alpha_{i}\right)$ are uniform. More generally, one can treat a discrete distribution without linearly spaced nodes and/or uniform masses.

3.2. Uniform distribution. The simplest continuous distribution is a uniform distribution. Consider

$$
d F(\tau)=\frac{1}{\tau_{b}-\tau_{a}} d \tau
$$

for $\tau_{a} \leq \tau \leq \tau_{b}$, and zero otherwise. Since the distribution function is constant, we may pull it outside the integral giving

$$
P(t, z)=\frac{1}{\tau_{b}-\tau_{a}} \int_{\tau_{a}}^{\tau_{b}} \mathcal{P}(t, z ; \tau) d \tau
$$

While this reduces to essentially integrating $\tau$ out of the polarization convolution term, for most polarization models this does not have an analytical solution. Therefore we must resort to numerical quadrature. Recall that the Composite Simpson rule approximation to

$$
I_{[a, b]}(L):=\int_{a}^{b} L(x) d x
$$


is

$$
S_{[a, b]}^{\ell}(L):=\sum_{i=0}^{\ell} c_{i}^{\ell} L\left(x_{i}\right) h
$$

where $\ell$ is even, $x_{i}=a+i h, h=\frac{b-a}{\ell}$, and the weights are given by

$$
c_{i}^{\ell}= \begin{cases}\frac{1}{3} & \text { if } i=0 \text { or } i=\ell \\ \frac{4}{3} & \text { else if } i \text { odd } \\ \frac{2}{3} & \text { else if } i \text { even. }\end{cases}
$$

Thus, our Composite Simpson approximation to (15) can be written

$$
\begin{aligned}
P(t, z) & =\frac{1}{\tau_{b}-\tau_{a}} \sum_{i=0}^{\ell} c_{i}^{\ell} \mathcal{P}\left(t, z ; \tau_{i}\right) \tau_{h} \\
& =\sum_{i=0}^{\ell} \alpha_{i} \mathcal{P}\left(t, z ; \tau_{i}\right)
\end{aligned}
$$

with

$$
\alpha_{i}=\frac{c_{i}^{\ell}}{\ell}
$$

since the numerator in $\tau_{h}=\left(\tau_{b}-\tau_{a}\right) / \ell$ cancels the constant in front of the integral. Note here that while our nodes are still linearly spaced, now our weights are nonuniform. We could have used a uniform discrete distribution to approximate the continuous one, which would have resulted in uniform weights, but the corresponding quadrature rule for $g(t, z ; \tau)$ would have only been $O\left(\tau_{h}\right)$. The Composite Simpson rule provides better accuracy for the same number of function evaluations.

3.3. Log-normal. A more realistic model for the distribution of Debye relaxation times is given by the log-normal distribution (see [24]). Therefore we consider the probability distribution defined by

$$
d F(\tau)=\frac{1}{\sqrt{2 \pi \sigma^{2}}} \frac{1}{\ln 10} \frac{1}{\tau} \exp \left(-\frac{(\log \tau-\mu)^{2}}{2 \sigma^{2}}\right) d \tau,
$$

which means that $\log \tau$ is normally distributed with mean $\mu$ and variance $\sigma^{2}$. The $\ln 10$ term appears because we are using base 10 logarithms. Thus

$$
P(t, z)=\int_{0}^{\infty} \mathcal{P}(t, z ; \tau) d F(\tau) \approx \int_{\tau_{a}}^{\tau_{b}} \mathcal{P}(t, z ; \tau) d F(\tau),
$$

for $\tau_{a}$ sufficiently close to zero and $\tau_{b}$ sufficiently large. Since $\log \tau$ is normally distributed, we choose our nodes based on a uniform discretization of $\log \tau$, denoted $\left\{\xi_{i}\right\}_{i=0}^{\ell}$, from $\log \tau_{a}:=\mu-6 \sigma$ to $\log \tau_{b}:=\mu+6 \sigma$, where $\tau_{a}$ and $\tau_{b}$ are thusly defined by their logarithms. In this way we reduce our support to a compact set which should be large enough to effectively approximate our integral. In order to use the Composite Simpson rule we 
prefer to have a uniform discretization of nodes. Therefore we first change variables so that the integral is in terms of $\xi=\log \tau$. First, let

$$
\hat{f}(\xi)=\frac{1}{\sqrt{2 \pi \sigma^{2}}} \exp \left(-\frac{(\log \tau-\mu)^{2}}{2 \sigma^{2}}\right)
$$

and note that

$$
d \xi=d\left(\frac{\ln \tau}{\ln 10}\right)=\frac{1}{\ln 10} \frac{1}{\tau} d \tau
$$

Then we have that

$$
P(t, z) \approx \int_{\tau_{a}}^{\tau_{b}} \mathcal{P}(t, z ; \tau) d F(\tau)=\int_{\mu-6 \sigma}^{\mu+6 \sigma} \mathcal{P}\left(t, z ; 10^{\xi}\right) \hat{f}(\xi) d \xi,
$$

where implicitly $d F=f(\tau) d \tau$ and $d \hat{F}=\hat{f}(\xi) d \xi$. Applying the Composite Simpson rule gives finally

$$
P(t, z) \approx \sum_{i=0}^{\ell} \alpha_{i} \mathcal{P}\left(t, z ; \tau_{i}\right),
$$

where we define $\tau_{i}=10^{\xi_{i}}$ and

$$
\alpha_{i}=c_{i}^{\ell} \hat{f}(\xi) \xi_{h}=\frac{c_{i}^{\ell} 12 \sigma}{\ell} \frac{1}{\sqrt{2 \pi \sigma^{2}}} \exp \left(-\frac{\left(\log \tau_{i}-\mu\right)^{2}}{2 \sigma^{2}}\right) .
$$

For this case our nodes are nonlinearly spaced in $\tau$ and our weights are nonuniform.

The method used in the bi-Gaussian case is derived in a similar fashion. Consider that the polarization is driven by two distinct mechanisms, one with a dielectric distribution determined by the mean $\mu_{1}$ and the standard deviation $\sigma_{1}$, and the other determined by the mean $\mu_{2}$ and the standard deviation $\sigma_{2}$. Then we define $F_{1}\left(\tau ; \mu_{1}, \sigma_{1}\right)$ and $F_{2}\left(\tau ; \mu_{2}, \sigma_{2}\right)$ as log-normal distributions as above, and let our macroscopic electric polarization be a function of some combination of these distributions (e.g., determined by the relative volume percentage of each of two substances in a material). Thus if $d F(\tau)=\beta_{1} d F_{1}(\tau)+$ $\beta_{2} d F_{2}(\tau)$, then we again have the representation (16). For the discretization, we prefer to apply the Composite Simpson rule to each distribution separately and then combine at the end. In other words,

$$
P(t, z) \approx \beta_{1} \sum_{i=0}^{\ell} \alpha_{i}^{1} \mathcal{P}\left(t, z ; \tau_{i}^{1}\right)+\beta_{2} \sum_{i=0}^{\ell} \alpha_{i}^{2} \mathcal{P}\left(t, z ; \tau_{i}^{2}\right),
$$

where $\left\{\alpha_{i}^{1}\right\}$ and $\left\{\tau_{i}^{1}\right\}$ are determined by $\mu_{1}$ and $\sigma_{1}$, and $\left\{\alpha_{i}^{2}\right\}$ and $\left\{\tau_{i}^{2}\right\}$ are determined by $\mu_{2}$ and $\sigma_{2}$, as explained above.

4. Inverse problem formulation. Our goal is to estimate the probability distribution function $(\mathrm{PDF}) F \in \mathcal{P}(\mathcal{T})$ of relaxation parameters in a given model of the polarization, where $\mathcal{P}(\mathcal{T})$ is the set of all PDFs on the admissible region $\mathcal{T}=\left[\tau_{1}, \tau_{2}\right] \subset(0, \infty)$, by using reflections of electromagnetic interrogating signals off a metallic backing of a dielectric material. To this end we attempt to minimize the difference between simulations and observations of time-domain data. In our formulation, the observations, $\hat{E}_{j}$, are of the electric field $E$ at discrete times $t_{j}$ taken at $z=0$. Each simulation is a solution of 
Maxwell's equations given in (12) using the polarization model (14) with candidate values for the distribution of relaxation parameters. We propose a nonstandard least-squares measurement (see [14] for a detailed justification) for the objective function, which is given by

$$
J(F)=\sum_{j}|| E\left(t_{j}, 0 ; F\right)|-| \hat{E}_{j}||^{2},
$$

where $E(\cdot, \cdot ; F)$ is the solution to (12) and (14) corresponding to the distribution $F$. Thus the inverse problem is to solve

$$
\min _{F \in \mathcal{P}(\mathcal{T})} J(F)
$$

5. Inverse problem results using a discrete distribution. Consider a discrete distribution with two atoms at $\tau_{1}$ and $\tau_{2}$. Essentially, we are decomposing the electric polarization into two components, each dependent on distinct relaxation times as follows:

$$
P=\alpha_{1} \mathcal{P}\left(t, z ; \tau_{1}\right)+\alpha_{2} \mathcal{P}\left(t, z ; \tau_{2}\right),
$$

where each $\mathcal{P}\left(\cdot, \cdot ; \tau_{i}\right)$ satisfies a Debye polarization equation with parameter $\tau_{i}$. For now we assume the proportions $\alpha_{1}$ and $\alpha_{2}=1-\alpha_{1}$ are known. Thus we are attempting to solve the following least squares optimization problem:

$$
\min _{\left(\tau_{1}, \tau_{2}\right)} \sum_{j}|| E\left(t_{j}, 0 ;\left(\tau_{1}, \tau_{2}\right)\right)|-| \hat{E}_{j}||^{2}
$$

where $\hat{E}_{j}$ is synthetic data generated using the true solution $\left(\tau_{1}^{*}, \tau_{2}^{*}\right)$ in our simulator (with a highly refined mesh), and $E\left(t_{j}, 0 ;\left(\tau_{1}, \tau_{2}\right)\right)$ depends on each $\tau_{i}$ through its dependence on $P$; see for example (12). Figure 2 depicts an example of the log of the objective function plotted versus the logs of $\tau_{1}$ and $\tau_{2}$ (using a frequency of $10^{11} \mathrm{~Hz}, \alpha_{1}=\alpha_{2}=.5$, $\tau_{1}^{*}=10^{-7.5}$ and $\left.\tau_{2}^{*}=10^{-7.8}\right)$.

5.1. Analysis of objective function. We can see clearly from the log surface plot in Figure 2 that there exists a relation for the relaxation times for which the corresponding simulations best match the data. We will refer to this relation as the "curve of best fit".

Note that the appearance of many local minima is due to the steep descent near the "curve of best fit" since the lattice points of the mesh used do not always lie near the curve. If we trace along this curve, as displayed in Figure 3, we see that there are actually two global minima, the exact solutions of $\log \left(\tau_{1}\right)=-7.5$ and $\log \left(\tau_{2}\right)=-7.8$, and since the proportion used in this case was $\alpha_{1}=\alpha_{2}=.5$, we also have the symmetric solution where $\tau_{1}$ and $\tau_{2}$ are swapped.

Unfortunately, the scale of these plots shows that the difference between the objective function at the minimizers and any other point on this curve in our parameter space is less than $4 \times 10^{-10}$. Therefore the exact minimizing parameters are not likely to be identifiable in a practical, experimental setting.

The equation for this "best fit curve" can be derived by combining equations (12) and (14). Note that

$$
\ddot{P}(t, z)=\int_{0}^{t} \ddot{\mathcal{G}}(t-s, z) E(s, z) d s+\mathcal{G}(0, z) \dot{E}(t, z)+\dot{\mathcal{G}}(0, z) E(t, z),
$$




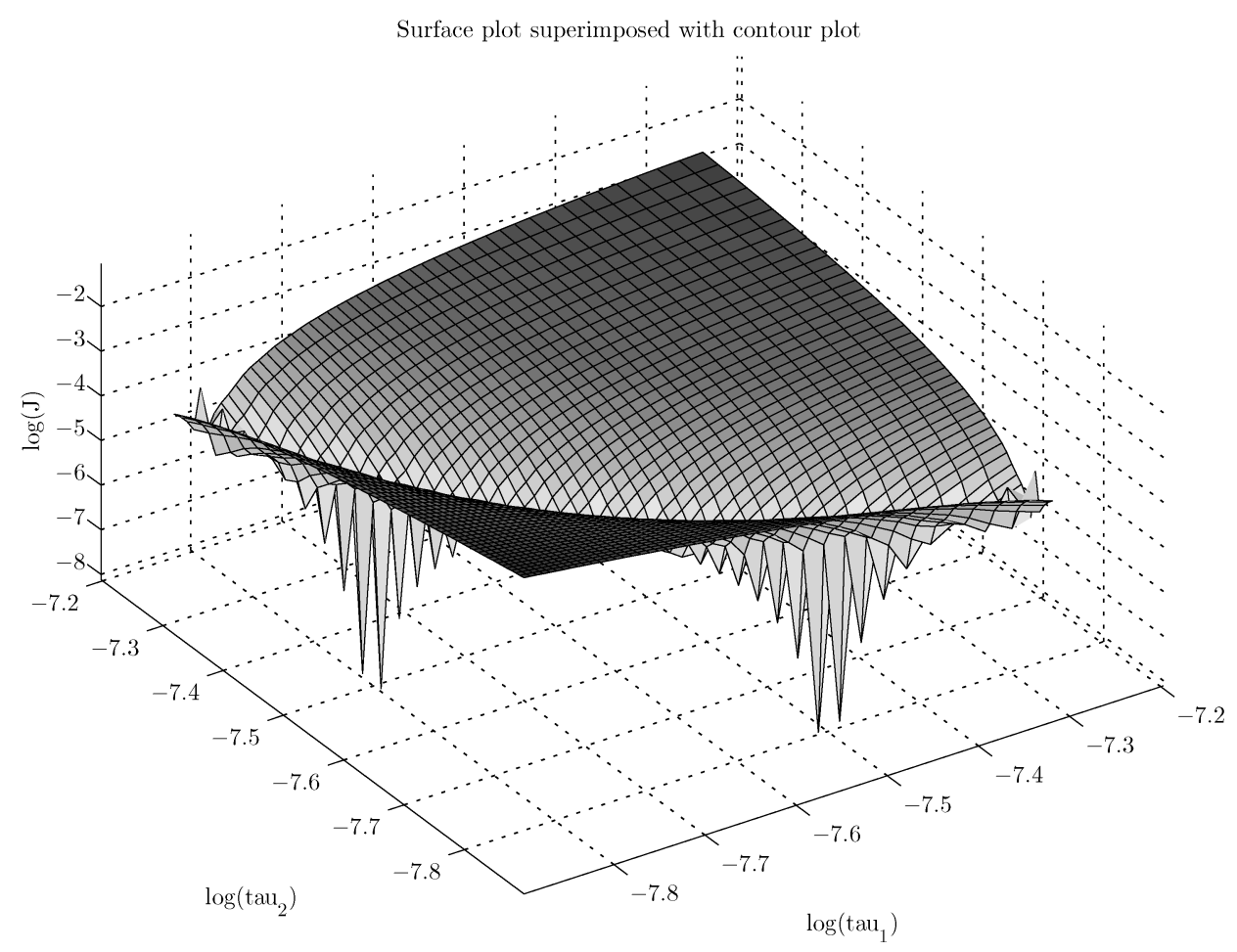

FIG. 2. The surface plot of the objective function for the relaxation time inverse problem superimposed with the contour plot along the curve of constant $\tilde{\lambda}$, using a frequency of $10^{11} \mathrm{~Hz}$.

where

$$
\ddot{\mathcal{G}}(t, z ; F)=\int_{\mathcal{T}} \ddot{g}(t, z ; \tau) d F(\tau),
$$

so that now substituting (20) into (12) we obtain

$$
\begin{aligned}
& \left\langle\epsilon_{r} \ddot{E}(t, \cdot), \phi\right\rangle+\left\langle I_{\Omega}\left[\eta_{0} \sigma+\mathcal{G}(0, z)\right] \dot{E}(t, \cdot), \phi\right\rangle+\left\langle I_{\Omega} \dot{\mathcal{G}}(0, \cdot) E(t, \cdot), \phi\right\rangle \\
& \quad+\left\langle\int_{0}^{t} I_{\Omega} \ddot{\mathcal{G}}(t-s, \cdot) E(s, \cdot) d s, \phi\right\rangle+\left\langle E^{\prime}(t, \cdot), \phi^{\prime}\right\rangle+\dot{E}(t, 0) \phi(0)=-\left\langle\eta_{0} \dot{J}_{s}(t, \cdot), \phi\right\rangle .
\end{aligned}
$$

For large frequencies, the $\dot{E}$ term in (21) dominates over all other terms which depend on $\mathcal{G}$. We can see this by considering the frequency domain where the time derivative causes an increase by a factor of $\omega$ in the norm. Thus the equation for the "curve of best fit" is simply that of constant $\mathcal{G}(0, z)$. For the discrete Debye example we have

$$
\mathcal{G}(0, z)=\int_{\mathcal{T}} g(0, z ; \tau) d F(\tau)=\alpha_{1} \epsilon_{0}\left(\epsilon_{s}-\epsilon_{\infty}\right) / \tau_{1}+\alpha_{2} \epsilon_{0}\left(\epsilon_{s}-\epsilon_{\infty}\right) / \tau_{2} .
$$

Therefore the equation is

$$
\frac{\alpha_{1}}{\tau_{1}}+\frac{\alpha_{2}}{\tau_{2}}=\frac{\alpha_{1}}{\tau_{1}^{*}}+\frac{\alpha_{2}}{\tau_{2}^{*}}
$$




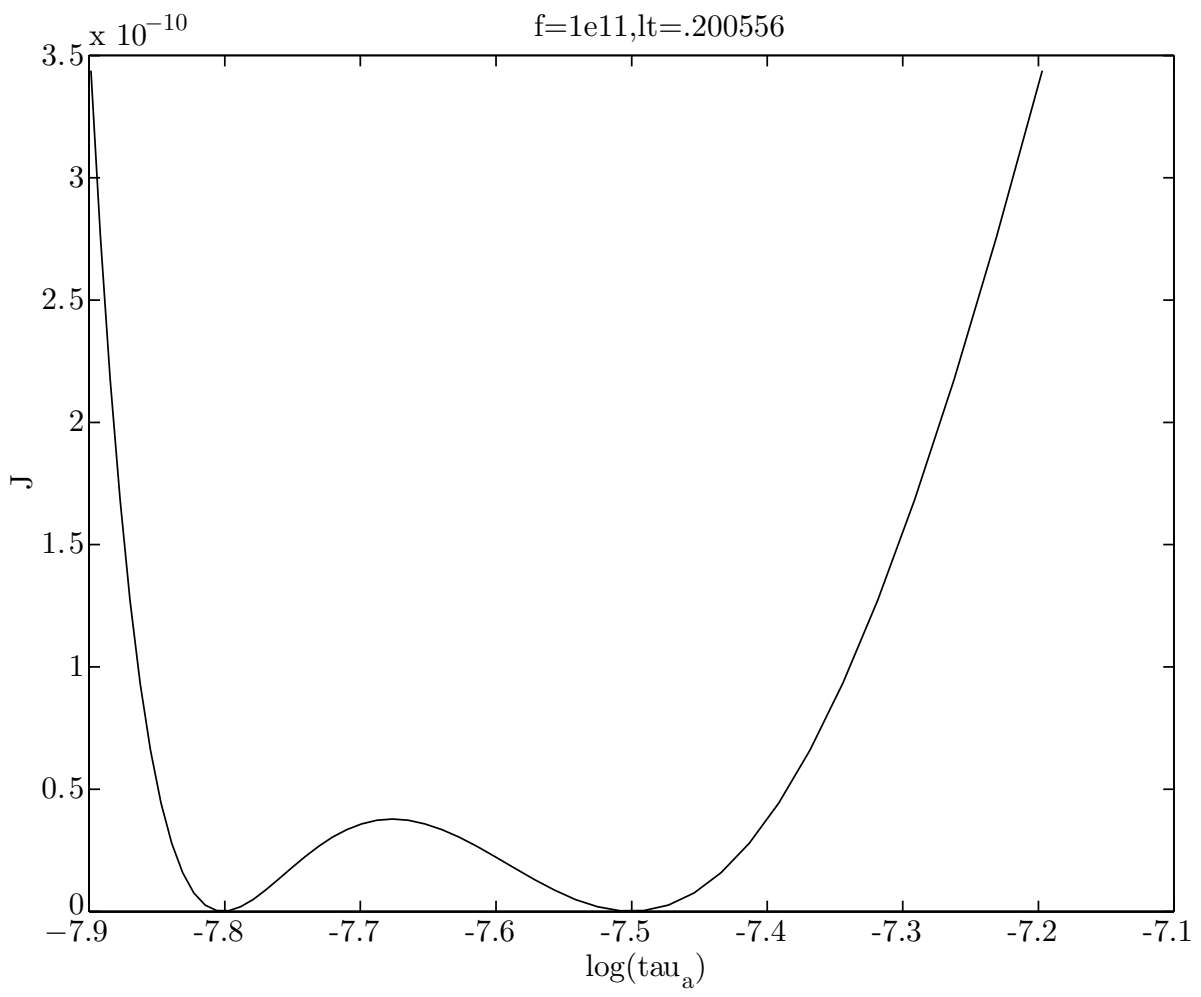

FIG. 3. The objective function for the relaxation time inverse problem plotted along the curve of constant $\tilde{\lambda}$ using a frequency of $10^{11} \mathrm{~Hz}$.

For scaling purposes, and to be consistent with the presentation in [11], we define

$$
\tilde{\lambda}:=\frac{1}{c \epsilon_{0}\left(\epsilon_{s}-\epsilon_{\infty}\right)} \mathcal{G}(0, z)
$$

which in this example means

$$
\tilde{\lambda}=\frac{\alpha_{1}}{c \tau_{1}}+\frac{\alpha_{2}}{c \tau_{2}}
$$

and thus we may say that the "curve of best fit" is the line of constant $\tilde{\lambda}$.

The frequency dependence of the $\dot{E}$ term in (21) suggests that for smaller frequencies it may not be the dominant contributor, and therefore, there may be a fundamentally different structure to the surface plot. This is in fact what we observe in our simulations. Figure 4 displays the $\log$ surface plot for $f=10^{6} \mathrm{~Hz}$. Note that the concavity of the "curve of best fit" has swapped orientation! This demonstrates that the surface plots are very much dependent on $\omega$ even though the relaxation mechanisms are the same. Computations for $f=10^{9} \mathrm{~Hz}$ were similar to $10^{11}$, and thus are not shown here. See [13] for those computational results.

Through our numerical calculations we have determined that for the case using a frequency of $10^{6} \mathrm{~Hz}$ the "curve of best fit" is actually that of constant $\tilde{\tau}:=\alpha_{1} \tau_{1}+$ $\alpha_{2} \tau_{2}$, which is what one might expect as this is the weighted average of the relaxation 


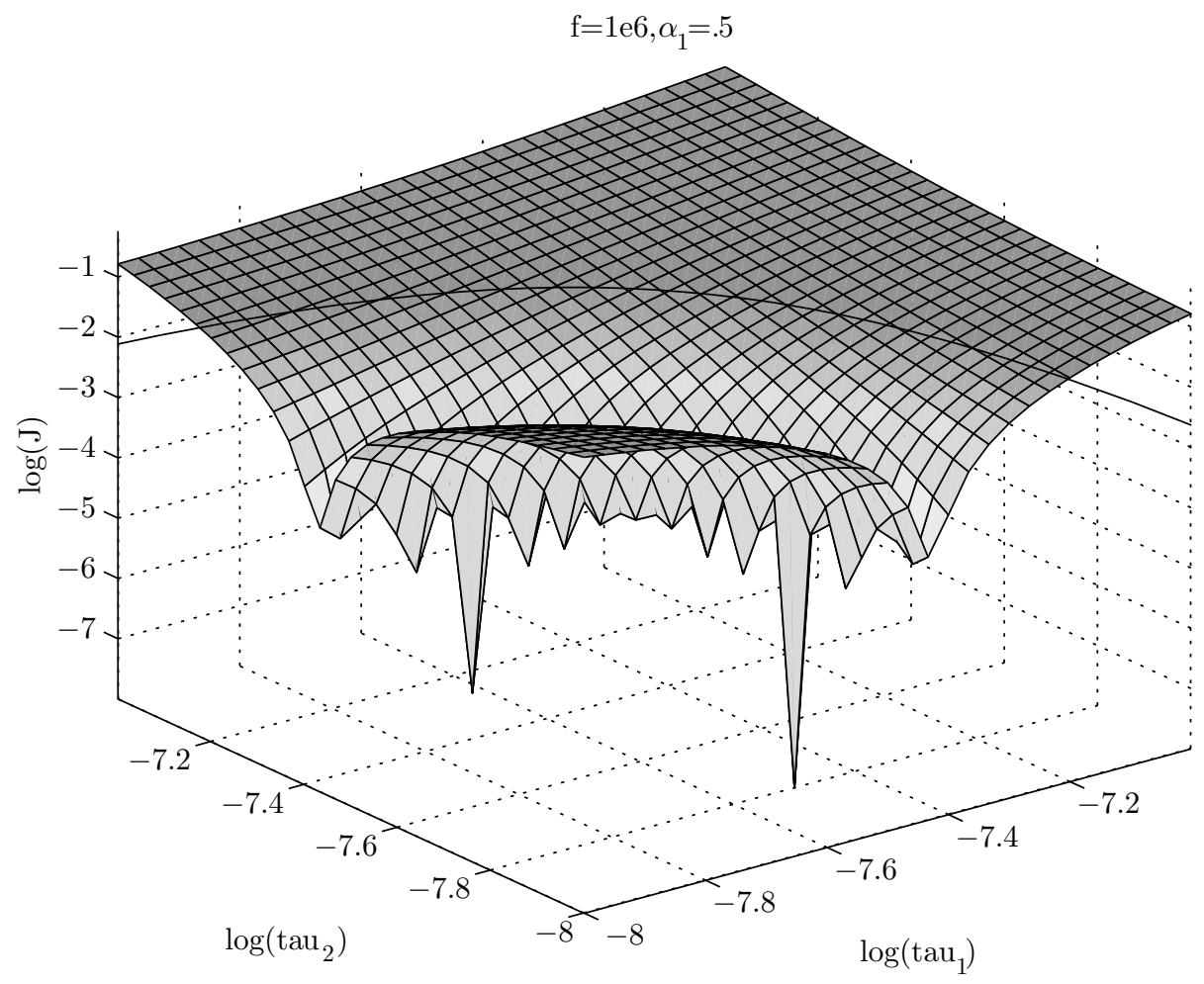

FIG. 4. The log of the objective function for the relaxation time inverse problem versus the $\log$ of $\tau_{1}$ and the $\log$ of $\tau_{2}$ using a frequency of $10^{6} \mathrm{~Hz}$. The solid line above the surface represents the curve of constant $\tilde{\tau}$.

times. For the example given here, $\tilde{\tau} \approx 2.37000 \times 10^{-8}$. In our simulations, angular frequencies less than one divided by this number were characterized by a constant $\tilde{\tau}$ while larger frequencies resulted in dominance by the $\tilde{\lambda}$ term given above. The fact that the regime characterized by $\omega \tau<1$ is fundamentally different in many respects from that of the $\omega \tau>1$ regime is well documented (see, for example, [24]). Still, the behavior of the objective function along its corresponding "curve of best fit" is similar for each frequency, despite the curves themselves being fundamentally different, as demonstrated by comparing Figure 3 to Figure 5 , which uses a frequency of $10^{6} \mathrm{~Hz}$. Note, however, that the scale of Figure 5 is several orders of magnitude larger, suggesting that the global minimizers may be easier to find for smaller frequencies.

REMARK 1. We should mention here that in order to analyze only the relationship between the interrogating frequency and the relaxation time, we scaled the dimensions of the interrogated object according to the change in scale of the frequency. For example, when the frequency is divided by 100000 to go from $10^{11}$ to $10^{6}$, we accordingly multiply the slab thickness by 100000 to get $400 \mathrm{~m}$ in order that the ratio of the dimensions to the wavelength of the signal remains the same. 


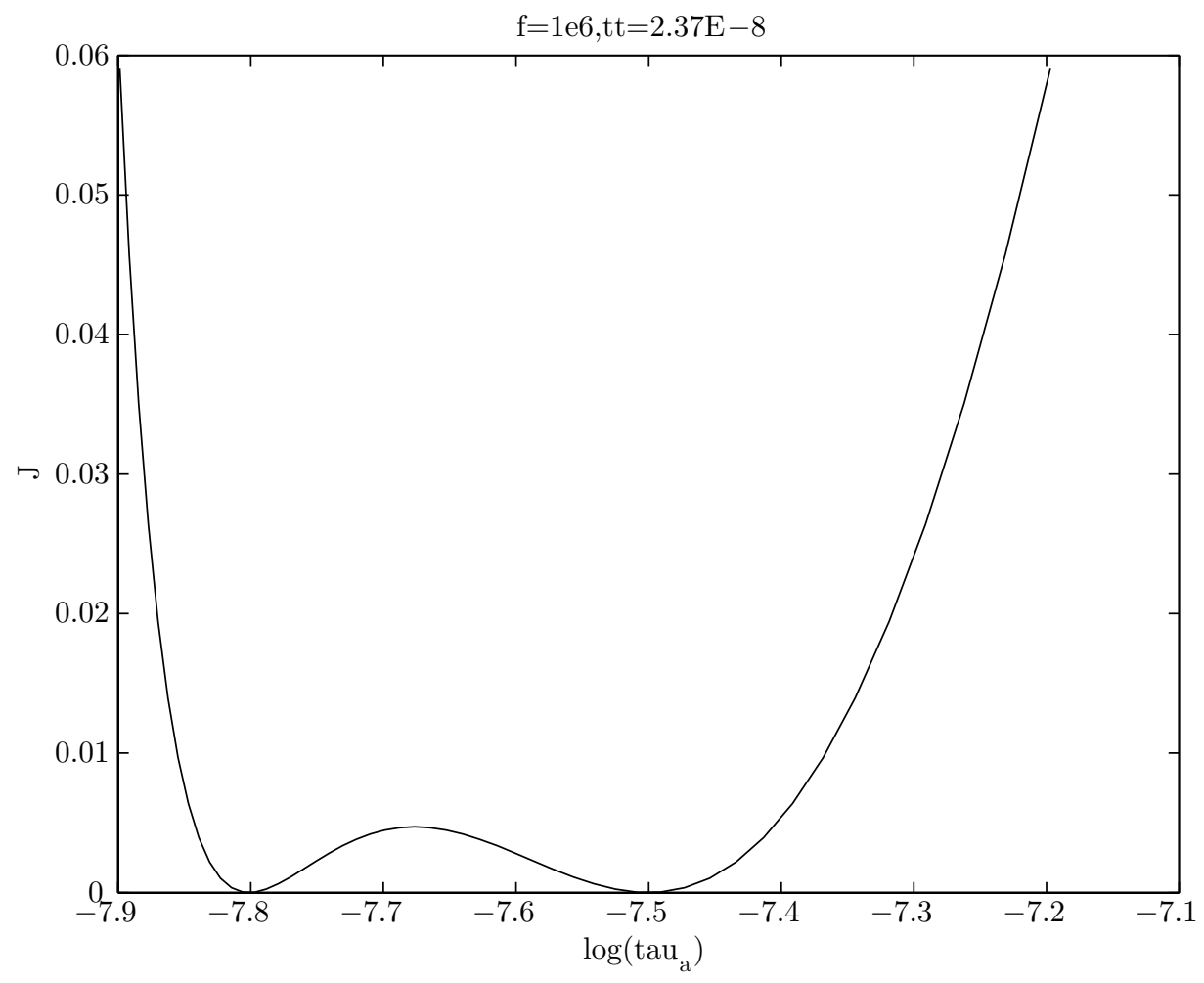

FIG. 5. The objective function for the relaxation time inverse problem plotted along the curve of constant $\tilde{\tau}$ using a frequency of $10^{6} \mathrm{~Hz}$.

5.2. Optimization procedure and results. We attempt to apply a two-parameter Levenberg-Marquardt optimization routine to the modified least squares error between the given data and our simulations, as defined in (17), to try to identify the two distinct relaxation times that generated the data. We are assuming that the corresponding volume proportions of the two materials $\left(\alpha_{1}\right.$ and $\left.\alpha_{2}:=1-\alpha_{1}\right)$ are known. We consider three different scenarios with respect to the volume proportions: $\alpha_{1} \in\{.1, .5, .9\}$. We also perform our inverse problem using the frequencies $10^{11} \mathrm{~Hz}$ and $10^{6} \mathrm{~Hz}$. Lastly we test the optimization procedure with three various initial conditions given in Table 1. The actual values are $\tau_{1}=10^{-7.50031} \approx 3.16 \times 10^{-8}$ and $\tau_{2}=10^{-7.80134} \approx 1.58 \times 10^{-8}$. Note that the first set of initial conditions is the farthest from the exact solution, while the third is the closest.

The results of the optimization are given in Tables 2 through 5 . Most of the cases appear not to have converged. Only a few of the cases corresponding to the closest initial conditions converged close to the original values of the relaxation times. But if we recall the shape of the objective function that we are trying to minimize (for example, see Figure 21), then it is understandable how a gradient based method may converge directly to the "curve of best fit". However, once it reaches this curve, the optimization routine 
may jump back and forth across the "trench" and may not be able to traverse the curve to find the true global minimum. This is in fact what is occurring.

TABLE 1. Three sets of initial conditions for the relaxation time inverse problem representing $\left(\tau_{1}^{0}, \tau_{2}^{0}\right)=\left(C \tau_{1}^{*}, \tau_{2}^{*} / C\right)$ for $C \in\{5,2,1.25\}$ respectively (case 0 represents the exact solution). Also given are the $\log _{10}$ of each relaxation time, as well as the $\%$ relative error from the exact value.

\begin{tabular}{|l|llllll|}
\hline case & $\tau_{1}$ & $\tau_{2}$ & $\log \left(\tau_{1}\right)$ & $\log \left(\tau_{2}\right)$ & $\% \tau_{1}$ & $\% \tau_{2}$ \\
\hline 0 & $3.1600 \mathrm{e}-8$ & $1.5800 \mathrm{e}-8$ & -7.50031 & -7.80134 & 0 & 0 \\
1 & $1.5800 \mathrm{e}-7$ & $3.1600 \mathrm{e}-9$ & -6.80134 & -8.50031 & 400 & 80 \\
2 & $6.3200 \mathrm{e}-8$ & $7.9000 \mathrm{e}-9$ & -7.19928 & -8.10237 & 100 & 50 \\
3 & $3.9500 \mathrm{e}-8$ & $1.2640 \mathrm{e}-8$ & -7.40340 & -7.89825 & 25 & 20 \\
\hline
\end{tabular}

TABLE 2. Resulting values of $\tau_{1}$ from the Levenberg-Marquardt routine using a frequency of $10^{11} \mathrm{~Hz}$ (recall the exact solution $\left.\tau_{1}^{*}=3.1600 \mathrm{e}-8\right)$.

\begin{tabular}{|l|lll|}
\hline & \multicolumn{3}{|c|}{$\alpha_{1}$} \\
case & .1 & .5 & .9 \\
\hline 1 & $1.57218 \mathrm{e}-07$ & $1.7488 \mathrm{e}-07$ & $2.26538 \mathrm{e}-07$ \\
2 & $6.34973 \mathrm{e}-08$ & $9.73445 \mathrm{e}-08$ & $4.90169 \mathrm{e}-08$ \\
3 & $3.98062 \mathrm{e}-08$ & $4.13321 \mathrm{e}-08$ & $3.43569 \mathrm{e}-08$ \\
\hline
\end{tabular}

TABLE 3. Resulting values of $\tau_{1}$ from the Levenberg-Marquardt routine using a frequency of $10^{6} \mathrm{~Hz}$ (recall the exact solution $\left.\tau_{1}^{*}=3.1600 \mathrm{e}-8\right)$.

\begin{tabular}{|l|lll|}
\hline & \multicolumn{3}{|c|}{$\alpha_{1}$} \\
case & .1 & .5 & .9 \\
\hline 1 & $3.79957 \mathrm{e}-08$ & $3.23393 \mathrm{e}-08$ & $3.16236 \mathrm{e}-08$ \\
2 & $3.17753 \mathrm{e}-08$ & $3.32218 \mathrm{e}-08$ & $3.21036 \mathrm{e}-08$ \\
3 & $3.17897 \mathrm{e}-08$ & $3.19001 \mathrm{e}-08$ & $3.16068 \mathrm{e}-08$ \\
\hline
\end{tabular}

Table 6 displays the values of $\tilde{\lambda}$ corresponding to each set of initial conditions, where case 0 denotes the exact solution. Table 7 displays the resulting values of $\tilde{\lambda}$ from the Levenberg-Marquardt routine using $f=10^{11} \mathrm{~Hz}$. Since smaller frequencies are expected to converge to the curve of constant $\tilde{\tau}$, we give the initial values of $\tilde{\tau}$ as well, in Table 8, and Table 9 displays the resulting values of $\tilde{\tau}$ after running the Levenberg-Marquardt routine using a frequency of $10^{6} \mathrm{~Hz}$. Note that while the initial values in case 1 are farthest from the exact solution, some of the corresponding $\tilde{\tau}$ are actually closest to the actual value (for example, $\alpha_{1}=.1$ ). While this suggests that this should more easily converge to the "curve of best fit", it will most likely be farther away from the exact solution on this curve, and therefore should still be considered the hardest of the cases to solve. 
TABLE 4. Resulting values of $\tau_{2}$ from the Levenberg-Marquardt routine using a frequency of $10^{11} \mathrm{~Hz}$ (recall the exact solution $\left.\tau_{2}^{*}=1.5800 \mathrm{e}-8\right)$.

\begin{tabular}{|l|lll|}
\hline & \multicolumn{3}{|c|}{$\alpha_{1}$} \\
case & .1 & .5 & .9 \\
\hline 1 & $1.51271 \mathrm{e}-08$ & $1.1208 \mathrm{e}-08$ & $3.2429 \mathrm{e}-09$ \\
2 & $1.53697 \mathrm{e}-08$ & $1.18119 \mathrm{e}-08$ & $6.10283 \mathrm{e}-09$ \\
3 & $1.56197 \mathrm{e}-08$ & $1.41322 \mathrm{e}-08$ & $1.1607 \mathrm{e}-08$ \\
\hline
\end{tabular}

TABLE 5. Resulting values of $\tau_{2}$ from the Levenberg-Marquardt routine using a frequency of $10^{6} \mathrm{~Hz}$ (recall the exact solution $\left.\tau_{2}^{*}=1.5800 \mathrm{e}-8\right)$.

\begin{tabular}{|l|lll|}
\hline & \multicolumn{3}{|c|}{$\alpha_{1}$} \\
case & .1 & .5 & .9 \\
\hline 1 & $1.51064 \mathrm{e}-08$ & $1.50634 \mathrm{e}-08$ & $1.63437 \mathrm{e}-08$ \\
2 & $1.57826 \mathrm{e}-08$ & $1.41845 \mathrm{e}-08$ & $1.12902 \mathrm{e}-08$ \\
3 & $1.57792 \mathrm{e}-08$ & $1.55032 \mathrm{e}-08$ & $1.57796 \mathrm{e}-08$ \\
\hline
\end{tabular}

Recognizing that we have converged to the "curve of best fit" in each of the above cases, we may now restart an optimization routine that traverses this curve. Some modifications to the optimization routine's parameters are required to address the vast difference in scales of this subproblem as compared to the two-parameter optimization problem. The final results of this two-step optimization approach are given in Tables 10 through 13. As expected, the results from Case 3 are generally better than the other two sets of initial conditions. The highest frequency attempted, $10^{11} \mathrm{~Hz}$, seemed to perform the most poorly (recall that $10^{9} \mathrm{~Hz}$ was also tested, and those results are reported in [13]). This suggests that the higher the frequency, the more difficult to accurately resolve the polarization mechanisms. Although the $10^{6} \mathrm{~Hz}$ case used the curve of constant $\tilde{\tau}$ while the $10^{9} \mathrm{~Hz}$ case used the curve of constant $\tilde{\lambda}$, there was no evidence to suggest that one case performed better than the other. Lastly, it appears that when material 1 (corresponding to the relaxation parameter $\tau_{1}$ ) is of the highest proportion, the optimization routine is best able to resolve $\tau_{1}$. Likewise, if material 1 is of a lower proportion, the routine instead does a better job of resolving $\tau_{2}$.

5.3. Determination of volume proportions. We now attempt to apply a one-parameter Levenberg-Marquardt optimization routine to our problem to identify the relative amounts of two materials with known, distinct relaxation times. Thus we are trying to find the corresponding volume proportions of the two materials $\left(\alpha_{1}\right.$ and $\left.\alpha_{2}:=1-\alpha_{1}\right)$. We again consider the three scenarios with respect to the exact volume proportions: $\alpha_{1} \in\{.1, .5, .9\}$. We also perform our inverse problem using the frequencies $10^{11} \mathrm{~Hz}$ and $10^{6} \mathrm{~Hz}$ (see 13 for $f=10^{9}$ ). Lastly we test the optimization procedure with three various initial conditions: $\alpha_{1}^{0} \in\{.9999, .0001, .5\}$ (except in the case when $\alpha_{1}^{*}=.5$, 
TABLE 6. The initial values of $\tilde{\lambda}:=\frac{\alpha_{1}}{c \tau_{1}}+\frac{\alpha_{2}}{c \tau_{2}}$ for each set of initial conditions (Case 0 represents the exact solution).

\begin{tabular}{|l|lll|}
\hline case & .1 & .5 & .9 \\
\hline 0 & 0.200556 & 0.158333 & 0.116111 \\
1 & 0.952112 & 0.538334 & 0.124556 \\
2 & 0.385278 & 0.237500 & 0.0897223 \\
3 & 0.245945 & 0.174167 & 0.102389 \\
\hline
\end{tabular}

TABLE 7. Resulting values of $\tilde{\lambda}$ from the Levenberg-Marquardt routine using a frequency of $10^{11} \mathrm{~Hz}$ for each set of initial conditions (case 0 represents the exact solution).

\begin{tabular}{|l|lll|}
\hline & \multicolumn{3}{|c|}{$\alpha_{1}$} \\
case & .1 & .5 & .9 \\
\hline 0 & 0.200556 & 0.158333 & 0.116111 \\
1 & 0.200573 & 0.15834 & 0.116109 \\
2 & 0.200573 & 0.158327 & 0.1159 \\
3 & 0.200573 & 0.158363 & 0.116114 \\
\hline
\end{tabular}

TABLE 8 . The initial values of $\tilde{\tau}:=\alpha_{1} \tau_{1}+\alpha_{2} \tau_{2}$ for each set of initial conditions (case 0 represents the exact solution).

\begin{tabular}{|l|lll|}
\hline case & .1 & .5 & .9 \\
\hline 0 & $1.7380 \mathrm{e}-08$ & $2.3700 \mathrm{e}-08$ & $3.0020 \mathrm{e}-08$ \\
1 & $1.8644 \mathrm{e}-08$ & $8.0580 \mathrm{e}-08$ & $1.42516 \mathrm{e}-07$ \\
2 & $1.3430 \mathrm{e}-08$ & $3.5550 \mathrm{e}-08$ & $5.7670 \mathrm{e}-08$ \\
3 & $1.5326 \mathrm{e}-08$ & $2.6070 \mathrm{e}-08$ & $3.6814 \mathrm{e}-08$ \\
\hline
\end{tabular}

TABLE 9. Resulting values of $\tilde{\tau}$ from the Levenberg-Marquardt routine using a frequency of $10^{6} \mathrm{~Hz}$ for each set of initial conditions (case 0 represents the exact solution).

\begin{tabular}{|l|lll|}
\hline & \multicolumn{3}{|c|}{$\alpha_{1}$} \\
case & .1 & .5 & .9 \\
\hline 0 & $1.7380 \mathrm{e}-08$ & $2.3700 \mathrm{e}-08$ & $3.0020 \mathrm{e}-08$ \\
1 & $1.73954 \mathrm{e}-08$ & $2.37014 \mathrm{e}-08$ & $3.00956 \mathrm{e}-08$ \\
2 & $1.73819 \mathrm{e}-08$ & $2.37031 \mathrm{e}-08$ & $3.00222 \mathrm{e}-08$ \\
3 & $1.73803 \mathrm{e}-08$ & $2.37016 \mathrm{e}-08$ & $3.00241 \mathrm{e}-08$ \\
\hline
\end{tabular}

in which case we used $\left.\alpha_{1}^{0} \in\{.9999, .0001, .4\}\right)$. We refer to these as Cases 1, 2, and 3 , respectively. In all of the following we assume that the known relaxation times are $\tau_{1}=10^{-7.50031} \approx 3.16 \times 10^{-8}$ and $\tau_{2}=10^{-7.80134} \approx 1.58 \times 10^{-8}$.

For each case the functions that we are attempting to minimize are well behaved and resemble quadratics (see [13] for plots). The results for this one-parameter inverse problem, displayed in Tables 14 and 15, verify that the relative proportions of known 
TABLE 10. Final estimates for $\tau_{1}$ from the two-step optimization approach using a frequency of $10^{11} \mathrm{~Hz}$ for each set of initial conditions (recall the exact solution $\tau_{1}^{*}=3.1600 \mathrm{e}-8$ ).

\begin{tabular}{|l|lll|}
\hline & \multicolumn{3}{|c|}{$\alpha_{1}$} \\
case & .1 & .5 & .9 \\
\hline 1 & $1.57221 \mathrm{e}-07$ & $2.81579 \mathrm{e}-08$ & $2.61108 \mathrm{e}-08$ \\
2 & $6.34951 \mathrm{e}-08$ & $4.63981 \mathrm{e}-08$ & $3.15232 \mathrm{e}-08$ \\
3 & $3.98043 \mathrm{e}-08$ & $3.33803 \mathrm{e}-08$ & $3.2833 \mathrm{e}-08$ \\
\hline
\end{tabular}

TABLE 11. Final estimates for $\tau_{1}$ from the two-step optimization approach using a frequency of $10^{6} \mathrm{~Hz}$ for each set of initial conditions (recall the exact solution $\tau_{1}^{*}=3.1600 \mathrm{e}-8$ ).

\begin{tabular}{|l|lll|}
\hline & \multicolumn{3}{|c|}{$\alpha_{1}$} \\
case & .1 & .5 & .9 \\
\hline 1 & $3.17673 \mathrm{e}-08$ & $3.16031 \mathrm{e}-08$ & $3.16986 \mathrm{e}-08$ \\
2 & $3.16206 \mathrm{e}-08$ & $3.16068 \mathrm{e}-08$ & $3.16031 \mathrm{e}-08$ \\
3 & $3.16031 \mathrm{e}-08$ & $3.16039 \mathrm{e}-08$ & $3.16053 \mathrm{e}-08$ \\
\hline
\end{tabular}

TABLE 12. Final estimates for $\tau_{2}$ from the two-step optimization approach using a frequency of $10^{11} \mathrm{~Hz}$ for each set of initial conditions (recall the exact solution $\tau_{2}^{*}=1.5800 \mathrm{e}-8$ ).

\begin{tabular}{|l|lll|}
\hline & \multicolumn{3}{|c|}{$\alpha_{1}$} \\
case & .1 & .5 & .9 \\
\hline 1 & $1.51273 \mathrm{e}-08$ & $1.68275 \mathrm{e}-08$ & $2.93245 \mathrm{e}-07$ \\
2 & $1.53699 \mathrm{e}-08$ & $1.36276 \mathrm{e}-08$ & $1.61391 \mathrm{e}-08$ \\
3 & $1.56196 \mathrm{e}-08$ & $1.53854 \mathrm{e}-08$ & $1.35142 \mathrm{e}-08$ \\
\hline
\end{tabular}

TABLE 13. Final estimates for $\tau_{2}$ from the two-step optimization approach using a frequency of $10^{6} \mathrm{~Hz}$ for each set of initial conditions (recall the exact solution $\tau_{2}^{*}=1.5800 \mathrm{e}-8$ ).

\begin{tabular}{|l|lll|}
\hline & \multicolumn{3}{|c|}{$\alpha_{1}$} \\
case & .1 & .5 & .9 \\
\hline 1 & $1.52384 \mathrm{e}-08$ & $1.52286 \mathrm{e}-08$ & $1.61666 \mathrm{e}-08$ \\
2 & $1.5787 \mathrm{e}-08$ & $1.45007 \mathrm{e}-08$ & $1.18861 \mathrm{e}-08$ \\
3 & $1.57845 \mathrm{e}-08$ & $1.55744 \mathrm{e}-08$ & $1.5783 \mathrm{e}-08$ \\
\hline
\end{tabular}

materials are generally easily identifiable. Tables 16 and 17 display the final objective function values for each case. Note that typical initial values for $J$ were around 0.1 ; furthermore, the tolerance was set at $10^{-9}$. Thus all but a few cases converged before reaching the maximum of 20 iterations. 
TABLE 14. Results for the one-parameter inverse problem to determine the relative proportion of two known Debye materials using a frequency of $10^{11} \mathrm{~Hz}\left(\alpha_{1}\right.$ estimates are shown).

\begin{tabular}{|l|lll|}
\hline & \multicolumn{3}{|c|}{$\alpha_{1}$} \\
case & .1 & .5 & .9 \\
\hline 1 & 0.10095 & 0.501353 & 0.900013 \\
2 & 0.0999994 & 0.5 & 0.9 \\
3 & 0.100643 & 0.499994 & 0.899994 \\
\hline
\end{tabular}

TABLE 15. Results for the one-parameter inverse problem to determine the relative proportion of two known Debye materials using a frequency of $10^{6} \mathrm{~Hz}$ ( $\alpha_{1}$ estimates are shown).

\begin{tabular}{|l|lll|}
\hline & \multicolumn{3}{|c|}{$\alpha_{1}$} \\
case & .1 & .5 & .9 \\
\hline 1 & 0.0995386 & 0.5 & 0.900008 \\
2 & 0.0999965 & 0.499969 & 0.899996 \\
3 & 0.100013 & 0.499999 & 0.899999 \\
\hline
\end{tabular}

TABLE 16. Final objective function values for the inverse problem to determine the relative proportion of two known Debye materials using a frequency of $10^{11} \mathrm{~Hz}$.

\begin{tabular}{|l|lll|}
\hline & \multicolumn{3}{|c|}{$\alpha_{1}$} \\
case & .1 & .5 & .9 \\
\hline 1 & $8.27897 \mathrm{e}-08$ & $1.69802 \mathrm{e}-07$ & $1.81577 \mathrm{e}-11$ \\
2 & $3.46217 \mathrm{e}-14$ & $1.64325 \mathrm{e}-18$ & $1.8982 \mathrm{e}-15$ \\
3 & $3.79016 \mathrm{e}-08$ & $3.76649 \mathrm{e}-12$ & $2.91698 \mathrm{e}-12$ \\
\hline
\end{tabular}

TABLE 17. Final objective function values for the inverse problem to determine the relative proportion of two known Debye materials using a frequency of $10^{6} \mathrm{~Hz}$.

\begin{tabular}{|l|lll|}
\hline & \multicolumn{3}{|c|}{$\alpha_{1}$} \\
case & .1 & .5 & .9 \\
\hline 1 & $7.74602 \mathrm{e}-08$ & $7.31725 \mathrm{e}-15$ & $4.39638 \mathrm{e}-12$ \\
2 & $4.51572 \mathrm{e}-12$ & $1.40031 \mathrm{e}-10$ & $1.03552 \mathrm{e}-12$ \\
3 & $6.11308 \mathrm{e}-11$ & $2.0608 \mathrm{e}-13$ & $1.53426 \mathrm{e}-13$ \\
\hline
\end{tabular}


5.4. Determination of volume proportions and relaxation times simultaneously. Although we anticipate this problem formulation to be underdetermined, we run our optimization routine for the problem where neither the relaxation times, nor the relative volume proportions, of two distinct Debye materials are known. Thus this is a threeparameter inverse problem for $\left\{\tau_{1}, \tau_{2}, \alpha_{1}\right\}$ (since $\alpha_{2}=1-\alpha_{1}$ ). Again we consider the following scenarios with respect to the actual volume proportions: $\alpha_{1} \in\{.1, .5, .9\}$. We also perform our inverse problem using the frequencies $10^{11} \mathrm{~Hz}, 10^{9} \mathrm{~Hz}$ and $10^{6} \mathrm{~Hz}$. Lastly we test the optimization procedure with the three various initial conditions that were given in Table 1 .

Our initial condition for the volume distribution is $\alpha_{1}^{0}=.9999$, which means there is essentially only material 1 . We chose this initial condition mainly to see whether the optimization routine would try to compensate for the altered volume distribution by significantly changing the relaxation times and not sufficiently correcting the volume distribution. This is in fact what seems to have occurred.

Of the cases that converged, none converged to the correct solution. For example, the final values of $\alpha_{1}$ were all greater than .6 (although the estimates corresponding to a smaller $\alpha_{1}^{*}$ were on average less than the estimates corresponding to $\alpha_{1}^{*}=.9$ ). When looking at the final estimates for the relaxation times, however, there appears to be no rhyme nor reason. This is quite similar to what happened when we first tried to determine unknown relaxation times, but with a fixed volume proportion, in Section 5 . Thus we may expect that here again no single parameter, $\tau_{1}, \tau_{2}$, nor $\alpha_{1}$, may converge, but instead we may see convergence of a general relation involving them all, namely $\tilde{\lambda}:=\frac{\alpha_{1}}{c \tau_{1}}+\frac{\alpha_{2}}{c \tau_{2}}$ (or respectively $\tilde{\tau}:=\alpha_{1} \tau_{1}+\alpha_{2} \tau_{2}$ for angular frequencies less than $\frac{1}{\tilde{\tau}}$ ).

While the inverse problem was unable to accurately resolve the individual values $\tau_{1}$, $\tau_{2}$, and $\alpha_{1}$, in all but a few cases (particularly in the $10^{9} \mathrm{~Hz}$ scenario) the optimization routine did converge as well as to be expected to the "curve of best fit". See [13] for tables of computations. In the previous section, with the fixed volume proportions, we were able to traverse the curve of constant $\tilde{\lambda}$ (or $\tilde{\tau}$ appropriately) to find the global minimum. However, here we truly have an underdetermined problem, and therefore we cannot extract any further information without providing more data.

5.5. Multiple frequency interrogation. In certain situations it may be feasible to add to the data used in the inverse problem in the previous section by interrogating the material again with a different frequency. In particular, if for example the first interrogating angular frequency is less than $\omega_{c}:=1 / \tilde{\tau}$, then choosing the second frequency to be greater than this critical value should give different information, i.e., $\omega_{L}<1 / \tilde{\tau}<\omega_{H}$. We have already seen that for frequencies lower than the critical angular frequency we are able to accurately resolve $\tilde{\tau}$. Thus, using this information we may choose a second frequency to be higher than the critical level, and therefore expect to be able to resolve $\tilde{\lambda}$, as was done in our previous examples for high frequencies. We applied this approach to the test cases described for the $f=10^{6} \mathrm{~Hz}$ interrogating signal. Using the results of the previous section, i.e., fixing $\tilde{\tau}$ and using the corresponding values of $\tau_{1}, \tau_{2}$, and $\alpha_{1}$ as initial conditions, we attempted an inverse problem to match data obtained by interrogating the same material with a frequency $f=10^{9} \mathrm{~Hz}$, which is higher than the critical value. In particular, we performed a one-parameter search for each of $\tau_{1}$ and $\tau_{2}$ 
successively, then a two-parameter search for both simultaneously for fine-tuning. As in previous inverse problems, this allowed determination of $\tilde{\lambda}$. Now with $\tilde{\lambda}$ and $\tilde{\tau}$ both fixed, we traverse along both contours simultaneously with a one-parameter inverse problem for $\tau_{1}$. Lastly, again, we do a full three-parameter inverse problem for fine-tuning. The results for Cases 1, 2, and 3 are given in Tables 18-20. Note that in order to ensure better accuracy when the wavelength is decreased, we double the number of finite elements used to solve the high frequency inverse problem.

In each case the lower frequency inverse problem was able to determine the value of $\tilde{\tau}$ to at least three decimal places, while the values of $\tau_{1}, \tau_{2}, \alpha_{1}$ and $\tilde{\lambda}$ are in general not necessarily even improved over the initial estimates (e.g., $\tau_{1}$ for Case 3 ). This is similar to the results of previous sections before traversing the contours of constant $\tilde{\tau}$ or $\tilde{\lambda}$. The higher frequency inverse problem with $\tilde{\tau}$ fixed was able to determine the value of $\tilde{\lambda}$ to at least three decimal places in each case, while again the values of $\tau_{1}, \tau_{2}$ and $\alpha_{1}$ are in general not necessarily even improved over the previous estimates. The final rows show the results from running a one-parameter inverse problem for $\tau_{1}$ with both $\tilde{\tau}$ and $\tilde{\lambda}$ fixed (and then using a three-parameter search for fine tuning). Each of the final estimates for $\tau_{1}, \tau_{2}$, and $\alpha_{1}$ have at least two decimal places accuracy.

Recall that, as explained in Remark 1 the slab thickness here is $400 \mathrm{~m}$. These dimensions are merely arbitrary examples, but there is a direct restriction on the width of a slab if it is to be reliably simultaneously interrogated with frequencies just above and just below the critical angular frequency for a given medium, which is described by the material properties through the value of $\tilde{\tau}$. Thus, for example, materials with relaxation times on the order of $10^{-11}$, like water, that have dimensions on the order of $.4 \mathrm{~m}$ are quite feasible to interrogate with multiple frequencies above and below its critical frequency.

6. Inverse problem results using a uniform distribution. The previous polarization inverse problems have assumed a discrete distribution with two atoms. According to experimental reports 24, most materials demonstrate polarization effects described by a range of relaxation times. Here we consider the simplest of distributions by exploring the possibility of a uniform distribution of relaxation times $(\tau)$ between a lower and upper limit ( $\tau_{a}$ and $\tau_{b}$ respectively). This presents us again with a two-parameter inverse problem, namely, to try to resolve the endpoints of the distribution used to generate the given data. Computationally we still approximate this distribution with discrete nodes, but instead of just one at each endpoint, we use $\ell=13$ uniformly distributed within the interval (recall Section 3.2). Note that we do not wish to restrict our optimization routine to search only for $\tau_{a}<\tau_{b}$. Therefore subsequently if $\tau_{a}>\tau_{b}$ it is assumed without loss of generality that $\tau_{b}$ is the lower limit of the distribution and $\tau_{a}$ is the upper limit.

Figure 6] depicts the log of the objective function for a frequency of $10^{11} \mathrm{~Hz}$. The solid line in Figure [6 is the curve of constant $\tilde{\lambda}$. Given a uniform distribution of relaxation times in a Debye medium, this parameter is given (analytically) by

$$
\tilde{\lambda}:=\frac{1}{c\left(\tau_{b}-\tau_{a}\right)} \int_{\tau_{a}}^{\tau_{b}} \frac{d \tau}{\tau}=\frac{\ln \tau_{b}-\ln \tau_{a}}{c\left(\tau_{b}-\tau_{a}\right)} .
$$


TABLE 18. Numerical results from the inverse problem using data from interrogating the same medium with one frequency above and one frequency below the critical angular frequency for that medium. Given are the $\tau_{1}, \tau_{2}$, and $\alpha_{1}$ values along with the relative error for $\tilde{\lambda}$ and $\tilde{\tau}$ for each step of the inverse problem: exact solution, initial conditions for Case 1, and the solutions from the low frequency inverse problem, from the high frequency inverse problem with fixed $\tilde{\tau}$, and finally from fixing both $\tilde{\tau}$ and $\tilde{\lambda}$.

\begin{tabular}{|l|lllll|}
\hline Case 1 & $\tau_{1}$ & $\tau_{2}$ & $\alpha_{1}$ & rel err $(\tilde{\lambda})$ & rel err $(\tilde{\tau})$ \\
\hline Exact & $3.16 \mathrm{e}-8$ & $1.58 \mathrm{e}-8$ & 0.1 & 0 & 0 \\
Initial & $1.58 \mathrm{e}-7$ & $3.16 \mathrm{e}-9$ & 0.9999 & 0.894221 & 8.09005 \\
$f=10^{6} \mathrm{~Hz}$ & $2.12522 \mathrm{e}-8$ & $8.82441 \mathrm{e}-9$ & 0.687957 & 0.126493 & $3.33717 \mathrm{e}-4$ \\
$f=10^{9} \mathrm{~Hz}$ & $1.82157 \mathrm{e}-8$ & $8.82198 \mathrm{e}-9$ & 0.910418 & $1.24653 \mathrm{e}-4$ & $3.33717 \mathrm{e}-4$ \\
Contour & $3.13819 \mathrm{e}-8$ & $1.57826 \mathrm{e}-8$ & 0.102596 & $4.98614 \mathrm{e}-5$ & $1.72612 \mathrm{e}-4$ \\
\hline
\end{tabular}

TABLE 19. Numerical results from the inverse problem using data from interrogating the same medium with one frequency above and one frequency below the critical angular frequency for that medium. Given are the $\tau_{1}, \tau_{2}$, and $\alpha_{1}$ values along with the relative error for $\tilde{\lambda}$ and $\tilde{\tau}$ for each step of the inverse problem: exact solution, initial conditions for Case 2 , and the solutions from the low frequency inverse problem, from the high frequency inverse problem with fixed $\tilde{\tau}$, and finally from fixing both $\tilde{\tau}$ and $\tilde{\lambda}$.

\begin{tabular}{|l|lllll|}
\hline Case 2 & $\tau_{1}$ & $\tau_{2}$ & $\alpha_{1}$ & $\operatorname{rel} \operatorname{err}(\tilde{\lambda})$ & $\operatorname{rel~} \operatorname{err}(\tilde{\tau})$ \\
\hline Exact & $3.16 \mathrm{e}-8$ & $1.58 \mathrm{e}-8$ & 0.1 & 0 & 0 \\
Initial & $6.32 \mathrm{e}-8$ & $7.9 \mathrm{e}-9$ & 0.9999 & 0.736658 & 2.63605 \\
$f=10^{6} \mathrm{~Hz}$ & $1.91221 \mathrm{e}-8$ & $8.40313 \mathrm{e}-9$ & 0.836785 & $5.08337 \mathrm{e}-2$ & $4.25777 \mathrm{e}-4$ \\
$f=10^{9} \mathrm{~Hz}$ & $1.81458 \mathrm{e}-8$ & $8.47912 \mathrm{e}-9$ & 0.920012 & $1.2964 \mathrm{e}-4$ & $4.25777 \mathrm{e}-4$ \\
Contour & $3.11405 \mathrm{e}-8$ & $1.57683 \mathrm{e}-8$ & 0.105044 & $5.48475 \mathrm{e}-5$ & $1.78366 \mathrm{e}-4$ \\
\hline
\end{tabular}

TABLE 20. Numerical results from the inverse problem using data from interrogating the same medium with one frequency above and one frequency below the critical angular frequency for that medium. Given are the $\tau_{1}, \tau_{2}$, and $\alpha_{1}$ values along with the relative error for $\tilde{\lambda}$ and $\tilde{\tau}$ for each step of the inverse problem: exact solution, initial conditions for Case 3 , and the solutions from the low frequency inverse problem, from the high frequency inverse problem with fixed $\tilde{\tau}$, and finally from fixing both $\tilde{\tau}$ and $\tilde{\lambda}$.

\begin{tabular}{|l|lllll|}
\hline Case 3 & $\tau_{1}$ & $\tau_{2}$ & $\alpha_{1}$ & $\operatorname{rel} \operatorname{err}(\tilde{\lambda})$ & $\operatorname{rel~err}(\tilde{\tau})$ \\
\hline Exact & $3.16 \mathrm{e}-8$ & $1.58 \mathrm{e}-8$ & 0.1 & 0 & 0 \\
Initial & $3.95 \mathrm{e}-8$ & $1.264 \mathrm{e}-9$ & 0.9999 & 0.578859 & 1.27257 \\
$f=10^{6} \mathrm{~Hz}$ & $1.81785 \mathrm{e}-8$ & $1.09823 \mathrm{e}-8$ & 0.887927 & $1.79102 \mathrm{e}-2$ & $4.60299 \mathrm{e}-4$ \\
$f=10^{9} \mathrm{~Hz}$ & $1.87871 \mathrm{e}-8$ & $1.09078 \mathrm{e}-8$ & 0.820402 & $1.09695 \mathrm{e}-4$ & $4.60299 \mathrm{e}-4$ \\
Contour & $3.12584 \mathrm{e}-8$ & $1.57761 \mathrm{e}-8$ & 0.103767 & $4.48752 \mathrm{e}-5$ & $1.49597 \mathrm{e}-4$ \\
\hline
\end{tabular}


Note that in computations we must use the same quadrature method to evaluate this integral as we do for $\mathcal{G}$ to ensure a correct correlation. Although the curve appears slightly different from the discrete distribution case in Figure 2, the fact that this objective function is also small along this curve suggests that this problem should behave similarly to the discrete distribution case in Section [5, Figure 7 depicts the log of the objective function for a frequency of $10^{6} \mathrm{~Hz}$. We notice that again the orientation of the "curve of best fit" is different from the higher frequency case. The solid line in Figure 7 is the curve of constant $\tilde{\tau}:=\int_{\tau_{a}}^{\tau_{b}} \tau d F(\tau)=\left(\tau_{b}-\tau_{a}\right) / 2$. Again, the fact that the $\tilde{\lambda}$ term only dominates the behavior when the interrogating frequency is greater than $\frac{1}{2 \pi \tilde{\tau}}$ is consistent with the discrete distribution case. Based on our previous experience with the discrete distribution, we anticipate that the two-parameter inverse problem will simply converge to the "line of best fit". Thus instead, we use an equivalent method to converge to this curve, namely minimizing over just one-parameter, $\tau_{a}$, leaving $\tau_{b}$ fixed at its initial value (the same values as those given as $\tau_{2}^{0}$ in Table 11). It should be noted that one may actually perform two of the one-parameter constrained optimizations, one in each of the directions $\tau_{a}$ and $\tau_{b}$, to allow for the possibility of the first direction not converging, for example, if $\tau_{a}^{0}, \tau_{b}^{0} \leq 10^{-9}$. In general, the second direction of the optimization requires only enough iterations to verify convergence, as the first direction has usually already converged to the "line of best fit". Using this "one-parameter at a time" approach, we still converge to the "line of best fit", but theoretically use half as many function evaluations as the two-parameter inverse problem, since we only compute one gradient at each step. (In practice, only a third as many function evaluations were actually needed to get the same order of accuracy as the two-parameter inverse problem.)

We performed the one-parameter inverse problem using the three initial condition cases described above in Table 1 and again using the frequencies $10^{11} \mathrm{~Hz}, 10^{9} \mathrm{~Hz}$ and $10^{6} \mathrm{~Hz}$. The $\tau_{a}$ estimates from running Levenberg-Marquardt on the modified least squares objective function are given in Table 21. Again, as in the discrete case, the values of the relaxation times do not appear to be converging to the correct solution. But we expect that the optimization routine is converging to the "curve of best fit". To test this we must look at the approximations to $\tilde{\lambda}$ and $\tilde{\tau}$.

TABLE 21. Resulting values of $\tau_{a}$ from the one-parameter LevenbergMarquardt routine for the inverse problem to determine the endpoints of a uniform distribution of relaxation times (recall the exact solution $\left.\tau_{a}^{*}=3.16000 \mathrm{e}-8\right)$.

\begin{tabular}{|l|l|lcl|}
\hline & Initial & \multicolumn{3}{|c|}{ Frequency $(\mathrm{Hz})$} \\
case & & $10^{11}$ & $10^{9}$ & $10^{6}$ \\
\hline 1 & $1.58000 \mathrm{e}-7$ & $5.41874 \mathrm{e}-8$ & $5.40910 \mathrm{e}-8$ & $3.59781 \mathrm{e}-8$ \\
2 & $6.32000 \mathrm{e}-8$ & $4.01846 \mathrm{e}-8$ & $4.01788 \mathrm{e}-8$ & $3.43510 \mathrm{e}-8$ \\
3 & $3.95000 \mathrm{e}-8$ & $3.41828 \mathrm{e}-8$ & $3.41820 \mathrm{e}-8$ & $3.27009 \mathrm{e}-8$ \\
\hline
\end{tabular}




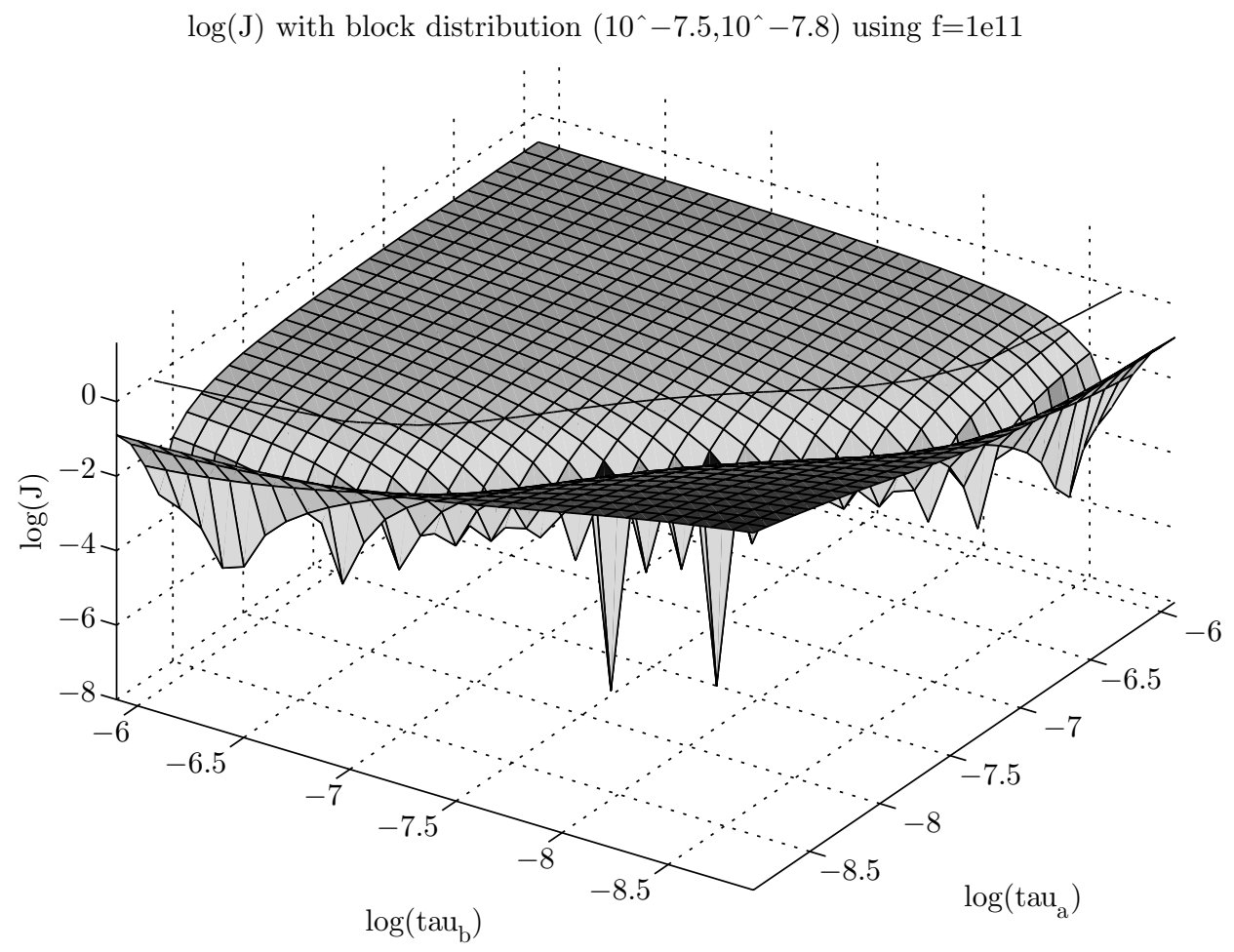

FIG. 6. The log of the objective function for the uniform distribution inverse problem versus the $\log$ of $\tau_{a}$ and the $\log$ of $\tau_{b}$ using a frequency of $10^{11} \mathrm{~Hz}$. The solid line above the surface represents the curve of constant $\tilde{\lambda}$.

The initial values of $\tilde{\lambda}$ and $\tilde{\tau}$ are given in Table 22 (note that these values were computed by $\tilde{\lambda} \approx \sum_{i} \frac{\alpha_{i}}{c \tau_{i}}$ and $\tilde{\tau} \approx \sum_{i} \alpha_{i} \tau_{i}$ using appropriately defined $\left\{\alpha_{i}\right\}_{i=0}^{N-1}$ determined by the Composite Simpson rule). The exact values of each are $\tilde{\lambda}^{*}=0.248369$ and $\tilde{\tau} *=5.42467 \times 10^{-8}$. The $\tilde{\lambda}$ and $\tilde{\tau}$ values resulting from running the one-parameter Levenberg-Marquardt routine are given in Table 23. Clearly each case has converged to the "line of best fit"; in general, the closer initial conditions converged closer to the actual value of $\tilde{\lambda}$ (or $\tilde{\tau}$ for $f=10^{6} \mathrm{~Hz}$ ).

After our one-parameter optimization routine resolved $\tilde{\lambda}$ ( or $\tilde{\tau}$ for $f=10^{6} \mathrm{~Hz}$ ), we minimized for $\tau_{a}$ along the line of constant $\tilde{\lambda}$ (or $\tilde{\tau}$ ). Again, this is a one-parameter inverse problem and therefore very efficient. The results of these computations are given in Tables 24 and 25, for $\tau_{a}$ and the corresponding $\tau_{b}$ (given constant $\tilde{\lambda}$ or $\tilde{\tau}$ ), respectively.

Finally, for fine tuning, we apply the full two-parameter Levenberg-Marquardt routine using the estimates from minimizing along constant $\tilde{\lambda}$ (or $\tilde{\tau}$ ). These results are shown in Tables 26 and 27. We see that the estimates change very little, if at all, which suggests that our approximation method is not only efficient, but quite accurate as well. 


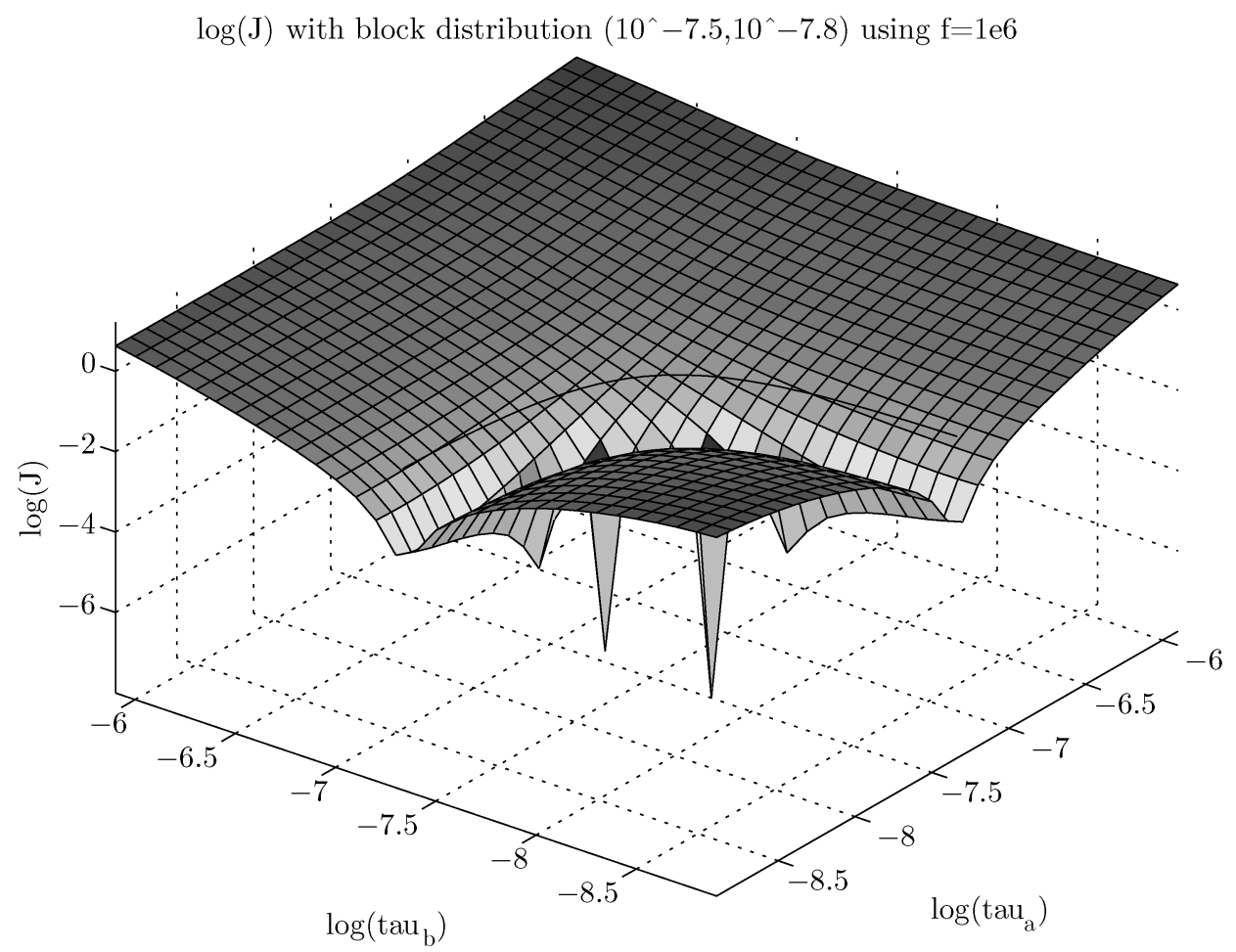

FIG. 7. The $\log$ of the objective function for the uniform distribution inverse problem versus the $\log$ of $\tau_{a}$ and the $\log$ of $\tau_{b}$ using a frequency of $10^{6} \mathrm{~Hz}$. The solid line above the surface represents the curve of constant $\tilde{\tau}$.

7. Inverse problem results using a Gaussian distribution. In this section we explore the possibility of determining dielectric parameters that are normally distributed. We apply a Gaussian distribution to the logarithms of the relaxation times (thus actually the relaxation times are log-normally distributed, but all of our computations are in "log"space, so we refer to these parameters as normally distributed). Based on experimental data in 24, this seems to be a likely model for the relaxation times of a material.

Recall from Section 3.3 that our PDF is

$$
d F(\tau ; \mu, \sigma)=\frac{1}{\sqrt{2 \pi \sigma^{2}}} \frac{1}{\ln 10} \frac{1}{\tau} \exp \left(-\frac{(\log \tau-\mu)^{2}}{2 \sigma^{2}}\right) d \tau
$$

for a log-normal distribution in $\tau$. Here $\mu$ is the mean of the relaxation times and $\sigma$ is the standard deviation. Recall further that the density is truncated to a support interval $\left[\tau_{a}, \tau_{b}\right]$ where $\tau_{a}$ and $\tau_{b}$ are determined based on $\mu$ and $\sigma$ as follows:

$$
\begin{aligned}
\tau_{a} & :=10^{\mu-6 \sigma}, \\
\tau_{b} & :=10^{\mu+6 \sigma} .
\end{aligned}
$$

Thus, this problem again presents us with a two-parameter inverse problem, namely, to try to resolve the mean and standard deviation of the distribution used to generate 
TABLE 22. The initial values of $\tilde{\lambda}:=\sum_{i} \frac{\alpha_{i}}{c \tau_{i}}$ (or $\tilde{\tau}:=\sum_{i} \alpha_{i} \tau_{i}$ for $f=10^{6}$ ) for each set of initial conditions for the inverse problem to determine the endpoints of a uniform distribution of relaxation times (Case 0 represents the exact solution).

\begin{tabular}{|l|ll|}
\hline & \multicolumn{2}{|l|}{ Frequency $(\mathrm{Hz})$} \\
case & $10^{11}$ and $10^{9}$ & $10^{6}$ \\
\hline 0 & 0.248369 & $5.42467 \mathrm{e}-8$ \\
1 & 0.115739 & $2.33313 \mathrm{e}-7$ \\
2 & 0.176705 & $9.66433 \mathrm{e}-8$ \\
3 & 0.223136 & $6.42533 \mathrm{e}-8$ \\
\hline
\end{tabular}

TABLE 23. Resulting values of $\tilde{\lambda}$ (or $\tilde{\tau}$ for $f=10^{6}$ ) from the Levenberg-Marquardt routine for the inverse problem to determine the endpoints of a uniform distribution of relaxation times for each set of initial conditions (Case 0 represents the exact solution). The values in parentheses denote the absolute value of the difference as the number of digits shown here would not sufficiently distinguish the approximations from the exact solution.

\begin{tabular}{|l|lll|}
\hline & & Frequency $(\mathrm{Hz})$ \\
case & $10^{11}$ & $10^{9}$ & $10^{6}$ \\
\hline 0 & 0.248369 & 0.248369 & $5.42467 \mathrm{e}-8$ \\
1 & $(1.64144 \mathrm{e}-8)$ & 0.248701 & $5.43479 \mathrm{e}-8$ \\
2 & $(1.51187 \mathrm{e}-9)$ & 0.248396 & $5.43315 \mathrm{e}-8$ \\
3 & $(5.12895 \mathrm{e}-11)$ & 0.248373 & $5.42813 \mathrm{e}-8$ \\
\hline
\end{tabular}

the given data. Learning from the success in the previous section, we expect to apply a three-step approach: one-parameter Levenberg-Marquardt for the mean $\mu$ (which we expect to converge to the line of constant $\tilde{\lambda}$ or $\tilde{\tau}$ ), then traverse the line of constant $\tilde{\lambda}$ or $\tilde{\tau}$ (which is again one-parameter), and finally, perform two-parameter LevenbergMarquardt for fine-tuning. We can see from the surface plot using $f=10^{11}$ shown in Figure 8 that we do again have a trench representing a "line of best fit", which does in fact have a single minimizer, but it appears to be independent of $\sigma$. The trench is actually slightly slanted, as can be seen in Figure 9 which zooms in on $\mu$, and in fact, for the lower frequencies the slant is indeed in the opposite direction. Therefore while at first glance of Figure 8 traversing a line of constant $\tilde{\lambda}$ or $\tilde{\tau}$ may seem unnecessary, it does actually give better results than simply minimizing in the $\sigma$ direction alone.

REMARK 2. Note that the slant does not appear to be a consequence of our quadrature rule. Changing the number of nodes used in our computations had no effect on the slope. Furthermore, increasing the support on which the integral was computed to $12 \sigma$ had no effect, e.g., on either side of $\mu$. While $\int \mathcal{G}(0, z)=\mu$ is actually independent of $\sigma$ we still expect $\sigma$ to affect the objective function in some other way, and this is in fact what is happening. Lastly, the apparent local minima in Figure 9 are again just an artifact of the grid points not falling exactly on the "line of best fit". 
TABLE 24. Resulting values of $\tau_{a}$ from minimizing along the line of constant $\tilde{\lambda}$ (or $\tilde{\tau}$ for $f=10^{6} \mathrm{~Hz}$ ) for the inverse problem to determine the endpoints of a uniform distribution of relaxation times (recall the exact solution $\left.\tau_{a}^{*}=3.16000 \mathrm{e}-8\right)$.

\begin{tabular}{|l|lll|}
\hline & \multicolumn{3}{|c|}{ Frequency $(\mathrm{Hz})$} \\
case & $10^{11}$ & $10^{9}$ & $10^{6}$ \\
\hline 1 & $3.08694 \mathrm{e}-08$ & $3.1653 \mathrm{e}-08$ & $3.17617 \mathrm{e}-08$ \\
2 & $3.16401 \mathrm{e}-08$ & $3.16043 \mathrm{e}-08$ & $3.17357 \mathrm{e}-08$ \\
3 & $3.15905 \mathrm{e}-08$ & $3.16007 \mathrm{e}-08$ & $3.16559 \mathrm{e}-08$ \\
\hline
\end{tabular}

TABLE 25. Resulting values of $\tau_{b}$ from minimizing along the line of constant $\tilde{\lambda}$ (or $\tilde{\tau}$ for $f=10^{6} \mathrm{~Hz}$ ), for the inverse problem to determine the endpoints of a uniform distribution of relaxation times (recall the exact solution $\left.\tau_{b}^{*}=1.5800 \mathrm{e}-8\right)$.

\begin{tabular}{|l|lll|}
\hline & \multicolumn{3}{|c|}{ Frequency $(\mathrm{Hz})$} \\
case & $10^{11}$ & $10^{9}$ & $10^{6}$ \\
\hline 1 & $1.68829 \mathrm{e}-08$ & $1.56448 \mathrm{e}-08$ & $1.55282 \mathrm{e}-08$ \\
2 & $1.57433 \mathrm{e}-08$ & $1.57874 \mathrm{e}-08$ & $1.55715 \mathrm{e}-08$ \\
3 & $1.58134 \mathrm{e}-08$ & $1.5798 \mathrm{e}-08$ & $1.57053 \mathrm{e}-08$ \\
\hline
\end{tabular}

TABLE 26. Resulting values of $\tau_{a}$ from the two-parameter LevenbergMarquardt routine for the inverse problem to determine the endpoints of a uniform distribution of relaxation times (recall the exact solution $\left.\tau_{a}^{*}=3.16000 \mathrm{e}-8\right)$.

\begin{tabular}{|l|lll|}
\hline & \multicolumn{3}{|c|}{ Frequency $(\mathrm{Hz})$} \\
case & $10^{11}$ & $10^{9}$ & $10^{6}$ \\
\hline 1 & $3.08694 \mathrm{e}-08$ & $3.15996 \mathrm{e}-08$ & $3.16014 \mathrm{e}-08$ \\
2 & $3.16401 \mathrm{e}-08$ & $3.16005 \mathrm{e}-08$ & $3.16008 \mathrm{e}-08$ \\
3 & $3.15905 \mathrm{e}-08$ & $3.16008 \mathrm{e}-08$ & $3.16002 \mathrm{e}-08$ \\
\hline
\end{tabular}

TABLE 27. Resulting values of $\tau_{b}$ from the two-parameter LevenbergMarquardt routine for the inverse problem to determine the endpoints of a uniform distribution of relaxation times (recall the exact solution $\left.\tau_{b}^{*}=1.58000 \mathrm{e}-8\right)$.

\begin{tabular}{|l|lll|}
\hline & \multicolumn{3}{|c|}{ Frequency $(\mathrm{Hz})$} \\
case & $10^{11}$ & $10^{9}$ & $10^{6}$ \\
\hline 1 & $1.68829 \mathrm{e}-08$ & $1.58005 \mathrm{e}-08$ & $1.57988 \mathrm{e}-08$ \\
2 & $1.57433 \mathrm{e}-08$ & $1.57993 \mathrm{e}-08$ & $1.57991 \mathrm{e}-08$ \\
3 & $1.58134 \mathrm{e}-08$ & $1.57988 \mathrm{e}-08$ & $1.57998 \mathrm{e}-08$ \\
\hline
\end{tabular}




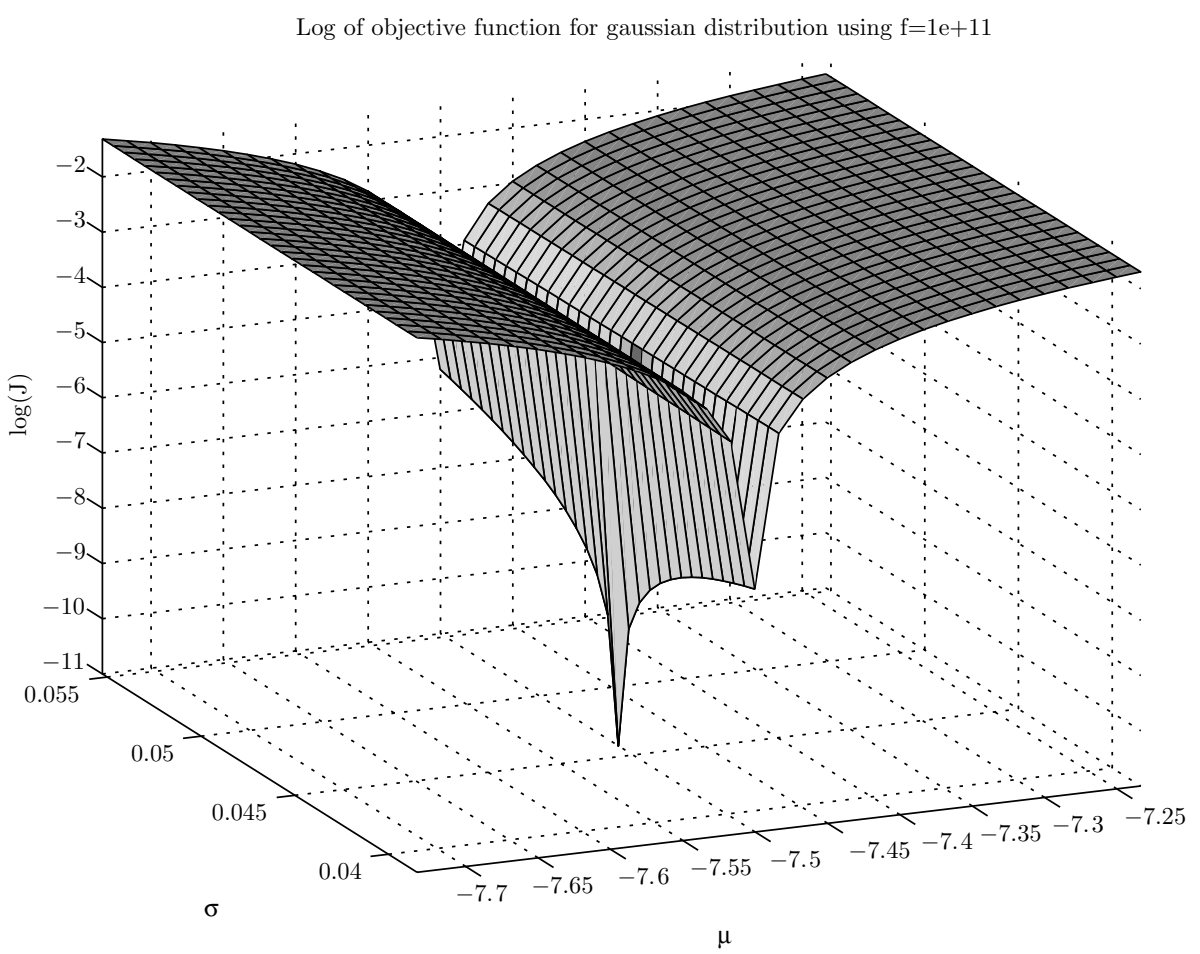

FIG. 8. The log of the objective function for the Gaussian distribution inverse problem versus the $\log$ of $\mu$ and $\sigma$ using a frequency of $10^{11} \mathrm{~Hz}$.

TABLE 28. Initial estimates and corresponding $\tilde{\lambda}$ and $\tilde{\tau}$ values for the inverse problem to determine the mean and standard deviation of a normal distribution of relaxation times (case 0 corresponds to the exact solution).

\begin{tabular}{|l|llll|}
\hline case & $\log \left(\mu_{0}\right)$ & $\sigma_{0}$ & $\tilde{\lambda}_{0}$ & $\tilde{\tau}_{0}$ \\
\hline 0 & -7.62525 & 0.0457575 & 0.141524 & $2.38319 \mathrm{e}-08$ \\
1 & -6.92628 & 0.036606 & 0.0282483 & $1.18922 \mathrm{e}-07$ \\
2 & -8.32422 & 0.054909 & 0.709351 & $4.77804 \mathrm{e}-09$ \\
3 & -7.62525 & 0.0411817 & 0.141375 & $2.37329 \mathrm{e}-08$ \\
\hline
\end{tabular}

We performed our inverse problem solution approach to the sample problem of $\mu=$ $10^{-7.62525}$ and $\sigma=0.0457575$ (this choice of parameters results in a distribution function comparable to the uniform distribution case of the previous section in that the corresponding densities have the same mean and roughly similar support, see Figure (10). The cases of initial values we considered for $\mu$ and $\sigma$, and the corresponding initial $\tilde{\lambda}$ and $\tilde{\tau}$ values, are given in Table 28. Note that Case 3 is specifically designed to test whether the standard deviation can be determined when the mean is known. 


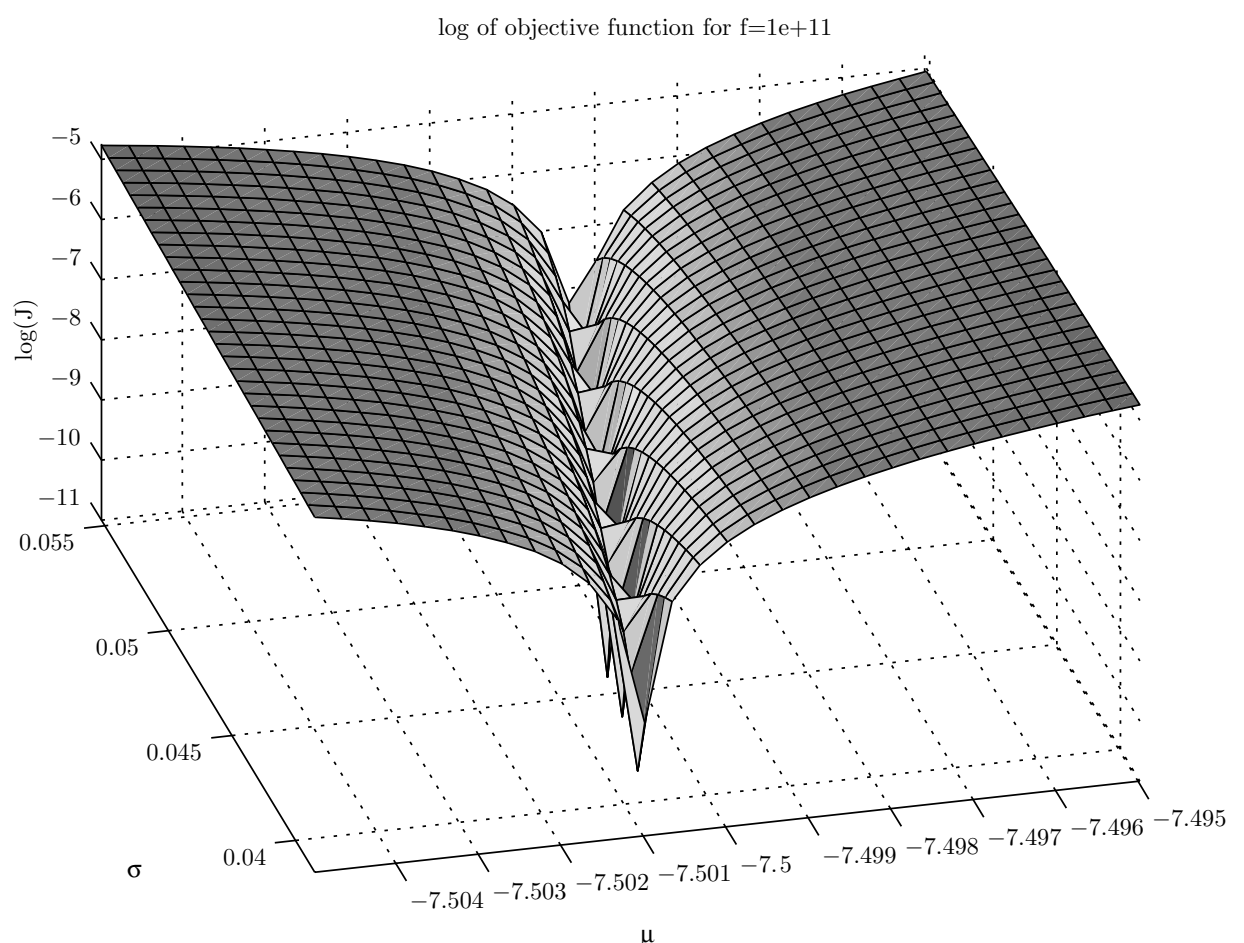

FIG. 9. The log of the objective function for the Gaussian distribution inverse problem versus the $\log$ of $\mu$ and $\sigma$, zoomed in with respect to $\mu$, and using a frequency of $10^{11} \mathrm{~Hz}$. The appearance of several local minima is merely an artifact of the lattice points used to plot the surface.

The $\mu$ estimates resulting from the one-parameter Levenberg-Marquardt are given in Table 29. In each case at least three decimal places of accuracy was achieved. Also, at least seven decimal places of accuracy are obtained on the estimates of the corresponding $\tilde{\lambda}$ and $\tilde{\tau}$ values, as displayed in Table 30 . The $\mu$ and $\sigma$ estimates resulting from the one-parameter tracing of the "line of best fit" are given in Tables 31 and 32, respectively. In each case at least six decimal places of accuracy were achieved for the log of $\mu$. Also, at least three decimal places of accuracy are obtained on the estimates of $\sigma$. Finally, the $\mu$ and $\sigma$ estimates resulting from the two-parameter Levenberg-Marquardt fine-tuning are given in Tables 33 and 34, respectively. In all but a couple cases we have as much degree accuracy as the number of digits shown here allows. Figure 11 gives a graphical representation of how well our inverse problem solution method converged given the initial condition Case 1 from Table 28. We conclude that the inverse problem involving a Gaussian distribution of relaxation times for a Debye polarization model is computationally feasible for the sample parameters presented here.

8. Inverse problem results using a bi-Gaussian distribution. In this section we combine the two ideas from Sections 5 and 7 in that we consider a material comprised 


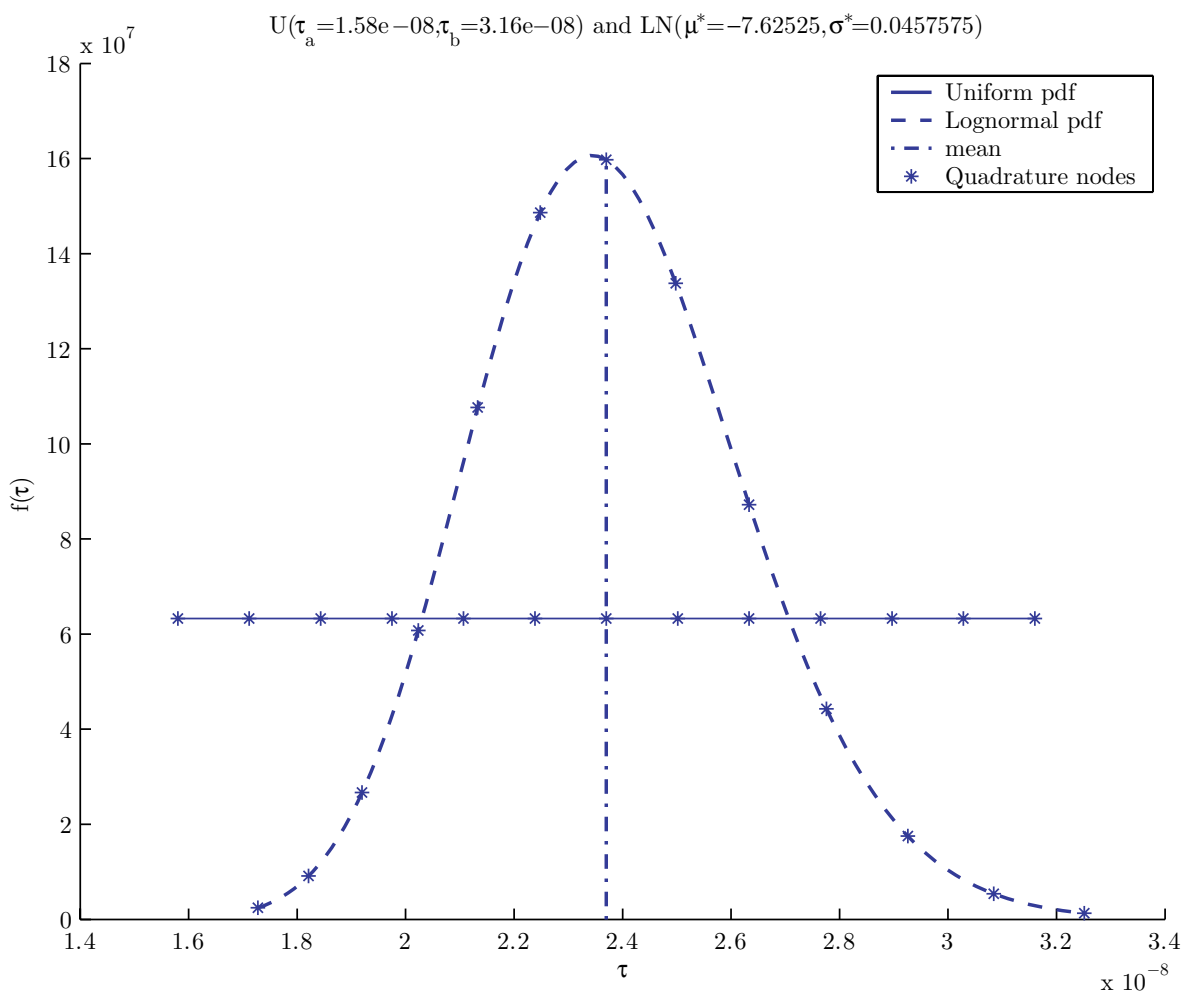

FIG. 10. This plot compares the uniform density considered in Section 6 to the log-normal density. Shown are the two density functions and the corresponding quadrature nodes used in integrating them.

of primarily two distinct polarization mechanisms, but rather than assuming two atoms at $\tau_{1}$ and $\tau_{2}$ as before we let $\mu_{1}=\tau_{1}$ and $\mu_{2}=\tau_{2}$ in two Gaussian distributions. Essentially, we are decomposing the distribution function into two components, each dependent on distinct means and standard deviations as follows:

$$
d \hat{F}=\alpha d F\left(\tau ; \mu_{1}, \sigma_{1}\right)+(1-\alpha) d F\left(\tau ; \mu_{2}, \sigma_{2}\right),
$$

where $d F$ is given by (24) (we take $\alpha=.5$ in our numerical experiments).

We now have a four-parameter inverse problem, namely

$$
\min _{\left(\mu_{1}, \sigma_{1}, \mu_{2}, \sigma_{2}\right) \in Q} J(\hat{F}),
$$

where $Q$ is the admissible region for our unknown parameters (e.g., $\sigma_{i}>0$ ). For the following examples, the true values of the means were taken to be $\left(\mu_{1}^{*}, \mu_{2}^{*}\right)=$ $\left(10^{-7.80134}, 10^{-7.50031}\right)$, with the standard deviations ranging from .02 to $.07 \mathrm{As}$ in the previous Gaussian case, we expect the objective function to be relatively less dependent on the standard deviations than the means; therefore, we address the dependency on the means first. Figure 12 shows a surface plot of the objective function with respect to $\mu_{1}$ and $\mu_{2}$. Here we can see the similarities to the distribution function from Section 5 , namely the presence of the "line of best fit" with two (symmetric) global minima. (When 
TABLE 29. The resulting $\log (\mu)$ values from performing a oneparameter Levenberg-Marquardt optimization for the inverse problem to determine the mean and standard deviation of a normal distribution of relaxation times for each frequency and initial value case (recall the exact solution is $\log \left(\mu^{*}\right)=-7.62525$ ).

\begin{tabular}{|l|lll|}
\hline & \multicolumn{3}{|c|}{ Frequency $(\mathrm{Hz})$} \\
case & $10^{11}$ & $10^{9}$ & $10^{6}$ \\
\hline 1 & -7.62612 & -7.6261 & -7.6244 \\
2 & -7.62419 & -7.62422 & -7.62629 \\
3 & -7.62571 & -7.62701 & -7.62348 \\
\hline
\end{tabular}

TABLE 30 . The corresponding $\tilde{\lambda}$ and $\tilde{\tau}$ values from performing a oneparameter Levenberg-Marquardt optimization for the inverse problem to determine the mean and standard deviation of a normal distribution of relaxation times for each frequency and initial value case. Note that the absolute values of the differences between the estimates and the exact values are shown as the number of digits shown here would not sufficiently distinguish the approximations from the exact solution.

\begin{tabular}{|l|lll|}
\hline & \multicolumn{3}{|c|}{ Frequency $(\mathrm{Hz})$} \\
case & $10^{11}$ & $10^{9}$ & $10^{6}$ \\
\hline 1 & $2.62469 \mathrm{e}-11$ & $7.38776 \mathrm{e}-08$ & $8.98789 \mathrm{e}-13$ \\
2 & $3.61986 \mathrm{e}-11$ & $9.10257 \mathrm{e}-08$ & $1.10726 \mathrm{e}-12$ \\
3 & $4.25993 \mathrm{e}-13$ & $1.56168 \mathrm{e}-07$ & $1.86751 \mathrm{e}-12$ \\
\hline
\end{tabular}

TABLE 31. The resulting $\log (\mu)$ values from traversing the "line of best fit" for the inverse problem to determine the mean and standard deviation of a normal distribution of relaxation times for each frequency and initial value case (recall the exact solution is $\log \left(\mu^{*}\right)=-7.62525$ ).

\begin{tabular}{|l|lll|}
\hline & \multicolumn{3}{|c|}{ Frequency $(\mathrm{Hz})$} \\
case & $10^{11}$ & $10^{9}$ & $10^{6}$ \\
\hline 1 & -7.62526 & -7.62525 & -7.62526 \\
2 & -7.62524 & -7.62525 & -7.62524 \\
3 & -7.62524 & -7.62525 & -7.62527 \\
\hline
\end{tabular}

comparing to Figure 2] note that here we have rotated the axis for better viewing of the minima under the surface.) Our minimization approach is thus the same as that which worked well in the previous sections. We perform a one-parameter Levenberg-Marquardt search in the $\mu_{1}$ direction (and just to be sure, a one-parameter Levenberg-Marquardt search in the $\mu_{2}$ direction), then optimize along the "line of best fit", and finally fine-tune with a two-parameter Levenberg-Marquardt search for both $\mu_{1}$ and $\mu_{2}$.

Thus far we have completely ignored the standard deviations. If we were to plot the objective function versus $\mu_{1}$ and $\mu_{2}$ for two distinct cases (using the exact values for 
TABLE 32. The resulting $\sigma$ values from traversing the "line of best fit" for the inverse problem to determine the mean and standard deviation of a normal distribution of relaxation times for each frequency and initial value case (recall the exact solution is $\sigma^{*}=0.0457575$ ).

\begin{tabular}{|l|lll|}
\hline & \multicolumn{3}{|c|}{ Frequency $(\mathrm{Hz})$} \\
case & $10^{11}$ & $10^{9}$ & $10^{6}$ \\
\hline 1 & 0.0456376 & 0.0457564 & 0.0456871 \\
2 & 0.0458453 & 0.0457592 & 0.0458442 \\
3 & 0.0458207 & 0.0457551 & 0.0456102 \\
\hline
\end{tabular}

TABLE 33. The resulting $\log (\mu)$ values from performing a two-parameter Levenberg-Marquardt optimization for fine-tuning of the solution for the inverse problem to determine the mean and standard deviation of a normal distribution of relaxation times for each frequency and initial value case (recall the exact solution is $\left.\log \left(\mu^{*}\right)=-7.62525\right)$.

\begin{tabular}{|l|lll|}
\hline & \multicolumn{3}{|c|}{ Frequency $(\mathrm{Hz})$} \\
case & $10^{11}$ & $10^{9}$ & $10^{6}$ \\
\hline 1 & -7.62526 & -7.62525 & -7.62525 \\
2 & -7.62524 & -7.62525 & -7.62525 \\
3 & -7.62524 & -7.62525 & -7.62525 \\
\hline
\end{tabular}

TABLE 34. The resulting $\sigma$ values from performing a two-parameter Levenberg-Marquardt optimization for fine-tuning of the solution for the inverse problem to determine the mean and standard deviation of a normal distribution of relaxation times for each frequency and initial value case (recall the exact solution is $\sigma^{*}=0.0457575$ ).

\begin{tabular}{|l|lll|}
\hline & \multicolumn{3}{|c|}{ Frequency $(\mathrm{Hz})$} \\
case & $10^{11}$ & $10^{9}$ & $10^{6}$ \\
\hline 1 & 0.0456376 & 0.0457576 & 0.0457575 \\
2 & 0.0458453 & 0.0457576 & 0.0457575 \\
3 & 0.0458207 & 0.0457575 & 0.0457575 \\
\hline
\end{tabular}

the standard deviations and using significantly incorrect ones), we would see that the location of the "line of best fit" does not change drastically (see [13]). This suggests that we should be able to at least determine the values of $\tilde{\lambda}$ or $\tilde{\tau}$ with just our one-parameter searches, as in the previous sections. However, the location of the local minima along this curve has changed; therefore, we should not expect our $\mu_{1}$ and $\mu_{2}$ estimates so far to be our final answer. We must first attempt to optimize with respect to the standard deviations, and then finally attempt a full four-parameter minimization to make sure we have accounted for all interdependencies. These are the last two-steps of our now sixstep optimization process for the four-parameter inverse problem involving a bi-Gaussian distribution.

The true values of the optimization parameters and the initial conditions considered for each case we attempted are given in Table 35. These values correspond to roughly 
TABLE 35. Initial estimates for the inverse problem to determine the means and standard deviations of a bi-Gaussian distribution of relaxation times. For each case, $\left(\mu_{1}^{*}, \mu_{2}^{*}\right)=\left(10^{-7.80134}, 10^{-7.50031}\right)$. For Case $1,\left(\sigma_{1}^{*}, \sigma_{2}^{*}\right)=(0.0457575,0.0457575)$. For Cases 2 and 3 , $\left(\sigma_{1}^{*}, \sigma_{2}^{*}\right)=(0.0705811,0.0222764)$.

\begin{tabular}{|l|llll|}
\hline case & $\log \left(\mu_{1}^{0}\right)$ & $\sigma_{1}^{0}$ & $\log \left(\mu_{2}^{0}\right)$ & $\sigma_{2}^{0}$ \\
\hline 1 & -8.50031 & 0.036606 & -6.80134 & 0.0571969 \\
2 & -7.10237 & 0.0846973 & -8.19928 & 0.0185637 \\
3 & -8.50031 & 0.0352905 & -6.80134 & 0.0445528 \\
\hline
\end{tabular}

TABLE 36 . The resulting $\log \left(\mu_{1}\right)$ values from performing a oneparameter Levenberg-Marquardt optimization in $\mu_{1}$ for the inverse problem to determine the means and standard deviations of a biGaussian distribution of relaxation times for each frequency and initial value case (recall the exact solution is $\log \left(\mu_{1}^{*}\right)=-7.80134$ ).

\begin{tabular}{|l|lll|}
\hline & \multicolumn{3}{|c|}{ Frequency $(\mathrm{Hz})$} \\
case & $10^{11}$ & $10^{9}$ & $10^{6}$ \\
\hline 1 & -7.94848 & -7.94847 & -8.37177 \\
2 & -7.10237 & -7.10237 & -7.39253 \\
3 & -7.95035 & -7.95034 & -8.37264 \\
\hline
\end{tabular}

TABLE 37. The resulting $\log \left(\mu_{2}\right)$ values from performing a oneparameter Levenberg-Marquardt optimization in $\mu_{2}$ for the inverse problem to determine the means and standard deviations of a biGaussian distribution of relaxation times for each frequency and initial value case (recall the exact solution is $\log \left(\mu_{2}^{*}\right)=-7.50031$ ).

\begin{tabular}{|l|lll|}
\hline & \multicolumn{3}{|c|}{ Frequency $(\mathrm{Hz})$} \\
case & $10^{11}$ & $10^{9}$ & $10^{6}$ \\
\hline 1 & -6.80134 & -6.80377 & -7.36944 \\
2 & -7.91764 & -7.91763 & -8.13781 \\
3 & -6.80134 & -6.80375 & -7.36803 \\
\hline
\end{tabular}

the same relative initial error as the initial value cases in the single Gaussian problem of Section 7 . The $\mu_{1}$ and $\mu_{2}$ estimates resulting from the one-parameter LevenbergMarquardt searches are given in Tables 36 and 37, respectively.

REMARK 3. Note that in Case 2 at $f=10^{11}$ the value of $\mu_{1}$ diverged toward zero in our first attempts. Therefore, the results shown in the tables for Case 2 at each frequency represent first minimizing in the $\mu_{2}$ direction followed by minimization in the $\mu_{1}$ direction. In general, better results are achieved when optimizing the smaller of the values (i.e., corresponding to a more negative logarithm) first.

Several of the $\mu_{2}$ estimates did not change from their initial values during the oneparameter optimization for $\mu_{2}$ suggesting that the $\mu_{1}$ search arrived sufficiently close to the $\tilde{\lambda}$ or $\tilde{\tau}$ curve (likewise for Case 2, the two higher frequency estimates for $\mu_{1}$ did not 


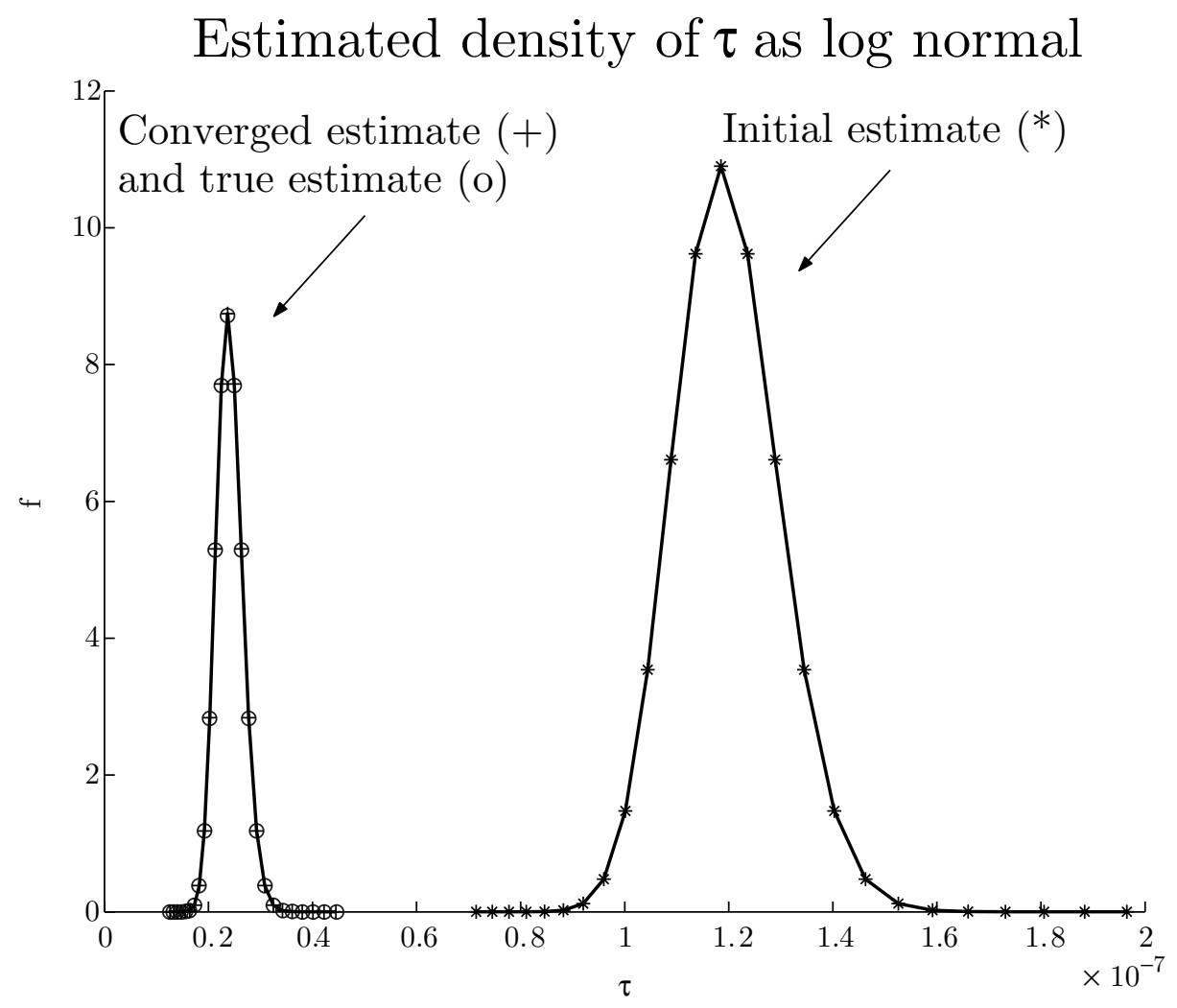

FIG. 11. Shown are the initial density function, the minimizing density function and the true density function (the latter two being practically identical) corresponding to Case 1 in Table 28

TABLE 38. The corresponding $\tilde{\lambda}$ and $\tilde{\tau}$ values from performing each one-parameter Levenberg-Marquardt optimization in $\mu_{1}$ and $\mu_{2}$ for the inverse problem to determine the means and standard deviations of a bi-Gaussian distribution of relaxation times for each frequency and initial value case. Note that the absolute values of the differences between the estimates and the exact values are shown.

\begin{tabular}{|l|lll|}
\hline & \multicolumn{3}{|c|}{ Frequency $(\mathrm{Hz})$} \\
case & $10^{11}$ & $10^{9}$ & $10^{6}$ \\
\hline 1 & $7.945 \mathrm{e}-10$ & $5.7109 \mathrm{e}-05$ & $1.45861 \mathrm{e}-10$ \\
2 & $5.00444 \mathrm{e}-10$ & $1.73813 \mathrm{e}-06$ & $4.24458 \mathrm{e}-10$ \\
3 & $1.74996 \mathrm{e}-08$ & $5.57019 \mathrm{e}-05$ & $1.46209 \mathrm{e}-10$ \\
\hline
\end{tabular}

change suggesting that the $\mu_{2}$ search converged sufficiently). The values of $\tilde{\lambda}$ or $\tilde{\tau}$ from the two one-parameter searches are given in Table 38. These values, on average, have about two fewer decimal places of accuracy as those in the single Gaussian case. Still, as the following results shall show, in general this is sufficiently close to allow for good final estimates. 


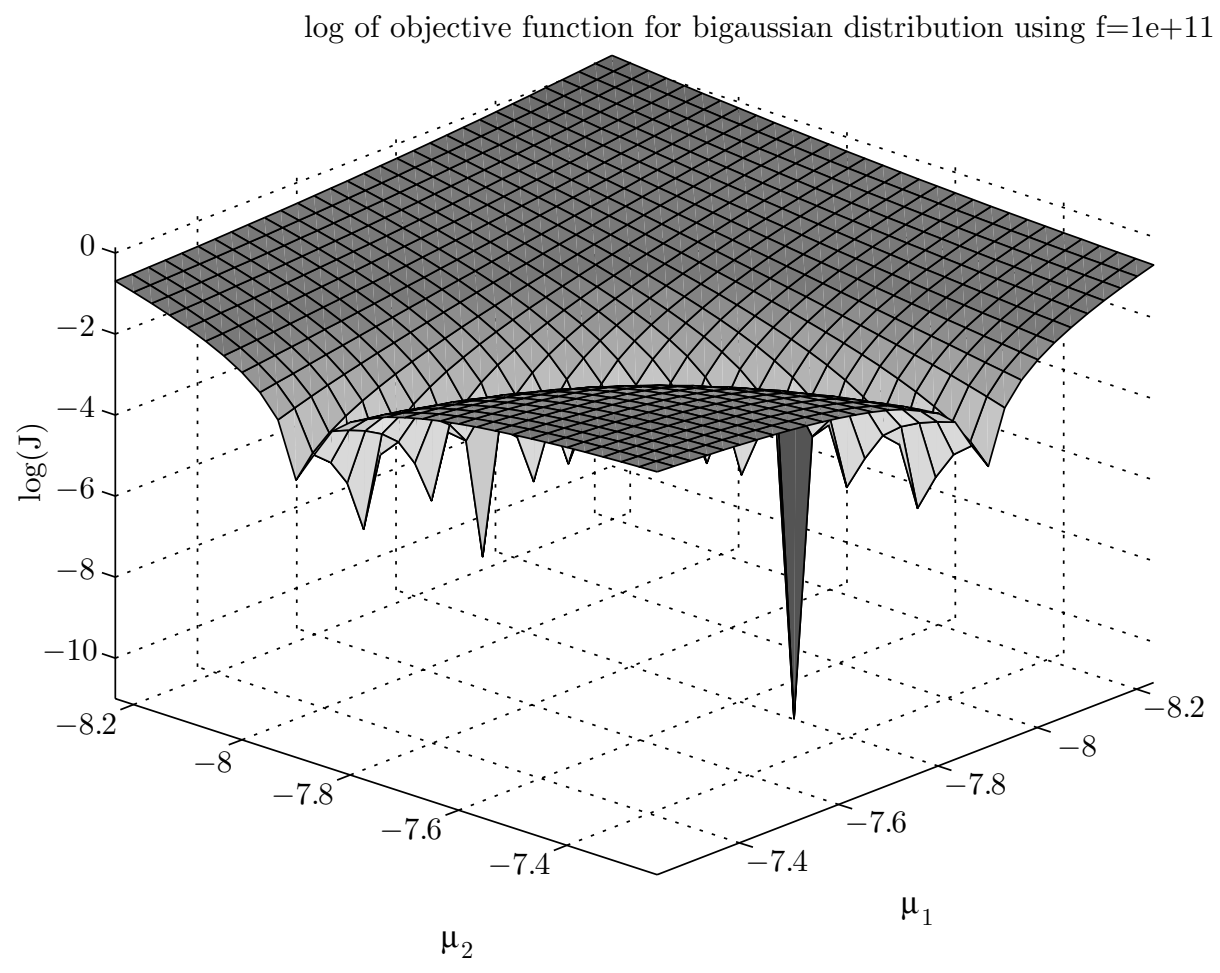

FIG. 12. The log of the objective function for the bi-Gaussian distribution inverse problem versus the log of $\mu_{1}$ and $\mu_{2}$ using a frequency of $10^{11} \mathrm{~Hz}$.

TABLE 39. The $\log \left(\mu_{1}\right)$ values resulting from traversing the "line of best fit" for the inverse problem to determine the mean and standard deviation of a normal distribution of relaxation times for each frequency and initial value case (recall the exact solution is $\left.\log \left(\mu_{1}^{*}\right)=-7.80134\right)$. The highlighted values denote convergence to the symmetric solution.

\begin{tabular}{|l|lll|}
\hline & \multicolumn{3}{|c|}{ Frequency $(\mathrm{Hz})$} \\
case & $10^{11}$ & $10^{9}$ & $10^{6}$ \\
\hline 1 & -7.80682 & $\mathbf{- 7 . 5 0 5 2}$ & -7.79549 \\
2 & $\mathbf{- 7 . 4 7 8 5 3}$ & $\mathbf{- 7 . 4 7 8 8 1}$ & $\mathbf{- 7 . 5 0 2 7 5}$ \\
3 & -7.81476 & $\mathbf{- 7 . 4 8 6 7 4}$ & -7.79517 \\
\hline
\end{tabular}

We now hold $\tilde{\lambda}$ or $\tilde{\tau}$ fixed and search for $\mu_{1}$ and $\mu_{2}$. The $\mu_{1}$ and $\mu_{2}$ estimates resulting from the one-parameter tracing of the "line of best fit" are given in Tables 39 and 40. respectively. Finally, the $\mu_{1}$ and $\mu_{2}$ estimates resulting from the two-parameter Levenberg-Marquardt fine-tuning are given in Tables 41 and 42, respectively. The lack of a significant difference after the two-parameter search suggests that we have found local minima with respect to $\mu_{1}$ and $\mu_{2}$ given our initial standard deviation estimates. 
TABLE 40. The $\log \left(\mu_{2}\right)$ values resulting from traversing the "line of best fit" for the inverse problem to determine the mean and standard deviation of a normal distribution of relaxation times for each frequency and initial value case (recall the exact solution is $\left.\log \left(\mu_{2}^{*}\right)=-7.50031\right)$. The highlighted values denote convergence to the symmetric solution.

\begin{tabular}{|l|lll|}
\hline & \multicolumn{3}{|c|}{ Frequency $(\mathrm{Hz})$} \\
case & $10^{11}$ & $10^{9}$ & $10^{6}$ \\
\hline 1 & -7.48957 & $\mathbf{- 7 . 7 9 7 7 5}$ & -7.50821 \\
2 & $\mathbf{- 7 . 8 1 2 9 9}$ & $\mathbf{- 7 . 8 1 2 8 5}$ & $\mathbf{- 7 . 7 8 2 4 6}$ \\
3 & -7.47935 & $\mathbf{- 7 . 8 1 0 6}$ & -7.50706 \\
\hline
\end{tabular}

TABLE 41. The $\log \left(\mu_{1}\right)$ values resulting from performing a twoparameter Levenberg-Marquardt optimization for fine-tuning of $\left(\mu_{1}, \mu_{2}\right)$ for the inverse problem to determine the means and standard deviations of a bi-Gaussian distribution of relaxation times for each frequency and initial value case (recall the exact solution is $\left.\log \left(\mu_{1}^{*}\right)=-7.80134\right)$. The highlighted values denote convergence to the symmetric solution.

\begin{tabular}{|l|lll|}
\hline & \multicolumn{3}{|c|}{ Frequency $(\mathrm{Hz})$} \\
case & $10^{11}$ & $10^{9}$ & $10^{6}$ \\
\hline 1 & -7.80682 & $\mathbf{- 7 . 5 0 5 2}$ & -7.79549 \\
2 & $\mathbf{- 7 . 4 7 8 5 1}$ & $\mathbf{- 7 . 4 7 8 8 1}$ & $\mathbf{- 7 . 5 0 2 7 5}$ \\
3 & -7.81442 & $\mathbf{- 7 . 4 8 6 7 4}$ & -7.79517 \\
\hline
\end{tabular}

TABLE 42. The $\log \left(\mu_{2}\right)$ values resulting from performing a twoparameter Levenberg-Marquardt optimization for fine-tuning of $\left(\mu_{1}, \mu_{2}\right)$ for the inverse problem to determine the means and standard deviations of a bi-Gaussian distribution of relaxation times for each frequency and initial value case (recall the exact solution is $\left.\log \left(\mu_{2}^{*}\right)=-7.50031\right)$. The highlighted values denote convergence to the symmetric solution.

\begin{tabular}{|l|lll|}
\hline & \multicolumn{3}{|c|}{ Frequency $(\mathrm{Hz})$} \\
case & $10^{11}$ & $10^{9}$ & $10^{6}$ \\
\hline 1 & $\mathbf{- 7 . 4 8 9 5 7}$ & $\mathbf{- 7 . 7 9 7 7 5}$ & -7.50821 \\
2 & $\mathbf{- 7 . 8 1 3}$ & $\mathbf{- 7 . 8 1 2 8 5}$ & $\mathbf{- 7 . 7 8 2 4 6}$ \\
3 & -7.47962 & $\mathbf{- 7 . 8 1 0 6}$ & -7.50706 \\
\hline
\end{tabular}

REMARK 4. Note that in some cases (actually, about half!) $\mu_{1}$ is converging toward $\mu_{2}^{*}$ (we have highlighted these cases in each subsequent table). This is the same problem that we encountered in the discrete case in Section [5. For our examples here, $\alpha=.5$, so 
TABLE 43. The $\sigma_{1}$ values resulting from a two-parameter LevenbergMarquardt search for $\sigma_{1}$ and $\sigma_{2}$ for the inverse problem to determine the means and standard deviations of a bi-Gaussian distribution of relaxation times for each frequency and initial value case (recall the exact solution is $\sigma_{1}^{*}=0.0457575$ for Case 1 and 0.0705811 otherwise). The highlighted values denote convergence to the symmetric solution.

\begin{tabular}{|l|lll|}
\hline & \multicolumn{3}{|c|}{ Frequency $(\mathrm{Hz})$} \\
case & $10^{11}$ & $10^{9}$ & $10^{6}$ \\
\hline 1 & 0.036606 & $\mathbf{0 . 0 0 6 0 3 3 1 1}$ & 0.0136791 \\
2 & $\mathbf{0 . 0 8 4 6 9 7 4}$ & $\mathbf{0 . 0 8 4 4 2 9 3}$ & $\mathbf{0 . 0 7 6 1 8 7 6}$ \\
3 & 0.0352905 & $\mathbf{0 . 0 0 8 5 7 0 5 8}$ & 0.0374784 \\
\hline
\end{tabular}

TABLE 44 . The $\sigma_{2}$ values resulting from a two-parameter LevenbergMarquardt search for $\sigma_{1}$ and $\sigma_{2}$ for the inverse problem to determine the means and standard deviations of a bi-Gaussian distribution of relaxation times for each frequency and initial value case (recall the exact solution is $\sigma_{2}^{*}=0.0457575$ for Case 1 and 0.0222764 otherwise). The highlighted values denote convergence to the symmetric solution.

\begin{tabular}{|l|lll|}
\hline & \multicolumn{3}{|c|}{ Frequency $(\mathrm{Hz})$} \\
case & $10^{11}$ & $10^{9}$ & $10^{6}$ \\
\hline 1 & 0.0571969 & $\mathbf{0 . 0 6 0 9 3 5}$ & 0.0658016 \\
2 & $\mathbf{0 . 0 1 8 5 6 3 7}$ & $\mathbf{0 . 0 1 9 2 0 2 3}$ & $\mathbf{0 . 0 4 4 2 8 8}$ \\
3 & 0.0445528 & $\mathbf{0 . 0 4 8 2 7 8 1}$ & 0.0456734 \\
\hline
\end{tabular}

TABLE 45. The $\log \left(\mu_{1}\right)$ values resulting from performing a fourparameter Levenberg-Marquardt optimization for all parameters in the inverse problem to determine the means and standard deviations of a bi-Gaussian distribution of relaxation times for each frequency and initial value case (recall the exact solution is $\log \left(\mu_{1}^{*}\right)=$ $-7.80134)$. The highlighted values denote convergence to the symmetric solution.

\begin{tabular}{|l|lll|}
\hline & \multicolumn{3}{|c|}{ Frequency $(\mathrm{Hz})$} \\
case & $10^{11}$ & $10^{9}$ & $10^{6}$ \\
\hline 1 & -7.80682 & $\mathbf{- 7 . 5 0 5 2}$ & -7.78543 \\
2 & $\mathbf{- 7 . 4 7 8 5 1}$ & $\mathbf{- 7 . 4 7 9 1 1}$ & $\mathbf{- 7 . 5 0 0 7 2}$ \\
3 & -7.81432 & $\mathbf{- 7 . 4 8 8 3 1}$ & -7.78507 \\
\hline
\end{tabular}

the problem is symmetric provided the standard deviations converge to the corresponding symmetric solution as well (i.e., $\sigma_{1}^{0}$ converges to $\sigma_{2}^{*}$ ). We could restrict our optimization problem to, for instance, only allow $\mu_{1}>\mu_{2}$, but we prefer the idea that there are two equally viable solutions to choose from since it may actually double our chances of finding one of them! If our initial estimates for each $\sigma_{i}$ were based on a certain ordering 
DISTRIBUTIONS OF DIELECTRIC MECHANISMS AND PARAMETERS

TABLE 46. The $\log \left(\mu_{2}\right)$ values resulting from performing a fourparameter Levenberg-Marquardt optimization for all parameters in the inverse problem to determine the means and standard deviations of a bi-Gaussian distribution of relaxation times for each frequency and initial value case (recall the exact solution is $\log \left(\mu_{2}^{*}\right)=$ $-7.50031)$. The highlighted values denote convergence to the symmetric solution.

\begin{tabular}{|l|lll|}
\hline & \multicolumn{3}{|c|}{ Frequency $(\mathrm{Hz})$} \\
case & $10^{11}$ & $10^{9}$ & $10^{6}$ \\
\hline 1 & -7.48957 & $\mathbf{- 7 . 7 9 6 8 7}$ & -7.50993 \\
2 & $\mathbf{- 7 . 8 1 3}$ & $\mathbf{- 7 . 8 1 2 7 9}$ & $\mathbf{- 7 . 8 0 6 9 1}$ \\
3 & -7.47506 & $\mathbf{- 7 . 8 0 9 9 2}$ & -7.50848 \\
\hline
\end{tabular}

TABLE 47. The $\sigma_{1}$ values resulting from a four-parameter LevenbergMarquardt search for all parameters in the inverse problem to determine the means and standard deviations of a bi-Gaussian distribution of relaxation times for each frequency and initial value case (recall the exact solution is $\sigma_{1}^{*}=0.0457575$ for Case 1 and 0.0705811 otherwise). The highlighted values denote convergence to the symmetric solution.

\begin{tabular}{|l|lll|}
\hline & \multicolumn{3}{|c|}{ Frequency $(\mathrm{Hz})$} \\
case & $10^{11}$ & $10^{9}$ & $10^{6}$ \\
\hline 1 & 0.0366059 & $\mathbf{0 . 0 0 5 6 3 1 2}$ & 0.0136811 \\
2 & $\mathbf{0 . 0 8 4 6 9 7 3}$ & $\mathbf{0 . 0 8 3 2 4 7 5}$ & $\mathbf{0 . 0 7 3 6 6 7 7}$ \\
3 & 0.0352905 & $\mathbf{0 . 0 0 8 7 9 7 2 5}$ & 0.037513 \\
\hline
\end{tabular}

TABLE 48. The $\sigma_{2}$ values resulting from a four-parameter LevenbergMarquardt search for all parameters in the inverse problem to determine the means and standard deviations of a bi-Gaussian distribution of relaxation times for each frequency and initial value case (recall the exact solution is $\sigma_{2}^{*}=0.0457575$ for Case 1 and 0.0222764 otherwise). The highlighted values denote convergence to the symmetric solution.

\begin{tabular}{|l|lll|}
\hline & \multicolumn{3}{|c|}{ Frequency $(\mathrm{Hz})$} \\
case & $10^{11}$ & $10^{9}$ & $10^{6}$ \\
\hline 1 & 0.0571968 & $\mathbf{0 . 0 6 0 4 7 9 3}$ & 0.0663628 \\
2 & $\mathbf{0 . 0 1 8 5 6 3 7}$ & $\mathbf{0 . 0 1 9 8 9 8 1}$ & $\mathbf{0 . 0 4 4 2 7 7 2}$ \\
3 & 0.0445528 & $\mathbf{0 . 0 4 7 7 3 4 8}$ & 0.0463262 \\
\hline
\end{tabular}

of the $\mu_{i}$ 's (for example, say we know the distribution of the smaller relaxation time is much more narrow) we could easily test for that ordering and rearrange our $\sigma_{i}$ estimates 
if necessary (though we would need to optimize with respect to each $\mu_{i}$ once more to reflect the changes).

While the results for determining the means of the unknown bi-Gaussian distribution are surprisingly decent, the optimization of the standard deviations was expectedly more difficult. Figure 13 demonstrates that the objective function behaves in a similar way with respect to the standard deviations as it did to the means in that there is a "line of best fit". Unfortunately, when compared to Figure 12 it becomes obvious that the scale involved in traversing along this curve is too small to be accurate enough for optimization. This is in fact what we experienced in attempting to minimize with respect to $\sigma_{1}$ along the "line of best fit". The changes in each $\sigma_{1}$ were in almost all cases less than six decimal places. Applying a two-parameter Levenberg-Marquardt for fine-tuning afterwards gave slightly better results for some cases, and we display these approximations in Tables 43 and 44. Using these values along with the best estimates so far for our means, we run a four-parameter Levenberg-Marquardt search. The results for this procedure are given in Tables 45 through 48.

One may argue that the lack of sensitivity to $\sigma$ may be due to starting initial estimates too close to the true solution, but this would not explain why, with all other parameters held fixed, gradient based methods take the standard deviation estimates farther from the true solution in half of the cases we tried. It is more likely that the numerical errors involved in the simulations are affecting the gradients. However, the results here reflect attempts that have been made to account for this using varying gradient step sizes (our Levenberg-Marquardt routine exits only after verifying small gradients with three different step sizes as explained in [14).

Our conclusion for the problem of determining the means and the standard deviations for a bi-Gaussian distribution of relaxation times is that the means are very readily determined with reasonable initial estimates, even with significantly incorrect standard deviations. However, the standard deviations are not easily determined even with quite accurate estimates for the means. This is most likely due to a lack of sensitivity of the resulting electromagnetic field to the variances in the bi-Gaussians.

9. Summary. In this report we have discussed theoretical and numerical results for modeling and estimation problems involving Maxwell's equations with a general polarization term which includes probability distributions for both dielectric parameters and mechanisms. A theoretical framework to treat such classes of problems was given along with stability results for the corresponding least squares inverse problems. We presented examples of distributions of parameters including discrete, uniform, log-normal, and logbi-Gaussian. In each case the objective function was characterized by a "line of best fit" corresponding to a curve of constant value of either $\lambda$ or $\tau$ depending on the relative values of the interrogating frequency $\omega$, and $1 / \tilde{\tau}$, where $\tilde{\tau}$ is the weighted average of the relaxation times. The constant values of these curves turned out to be, in fact, $\tilde{\tau}$ and $\tilde{\lambda}$, which is the weighted average of the inverses of the relaxation times (scaled by the speed of light for convenience). The similarities between the cases were exploited in determining effective optimization procedures for subsequent cases. 


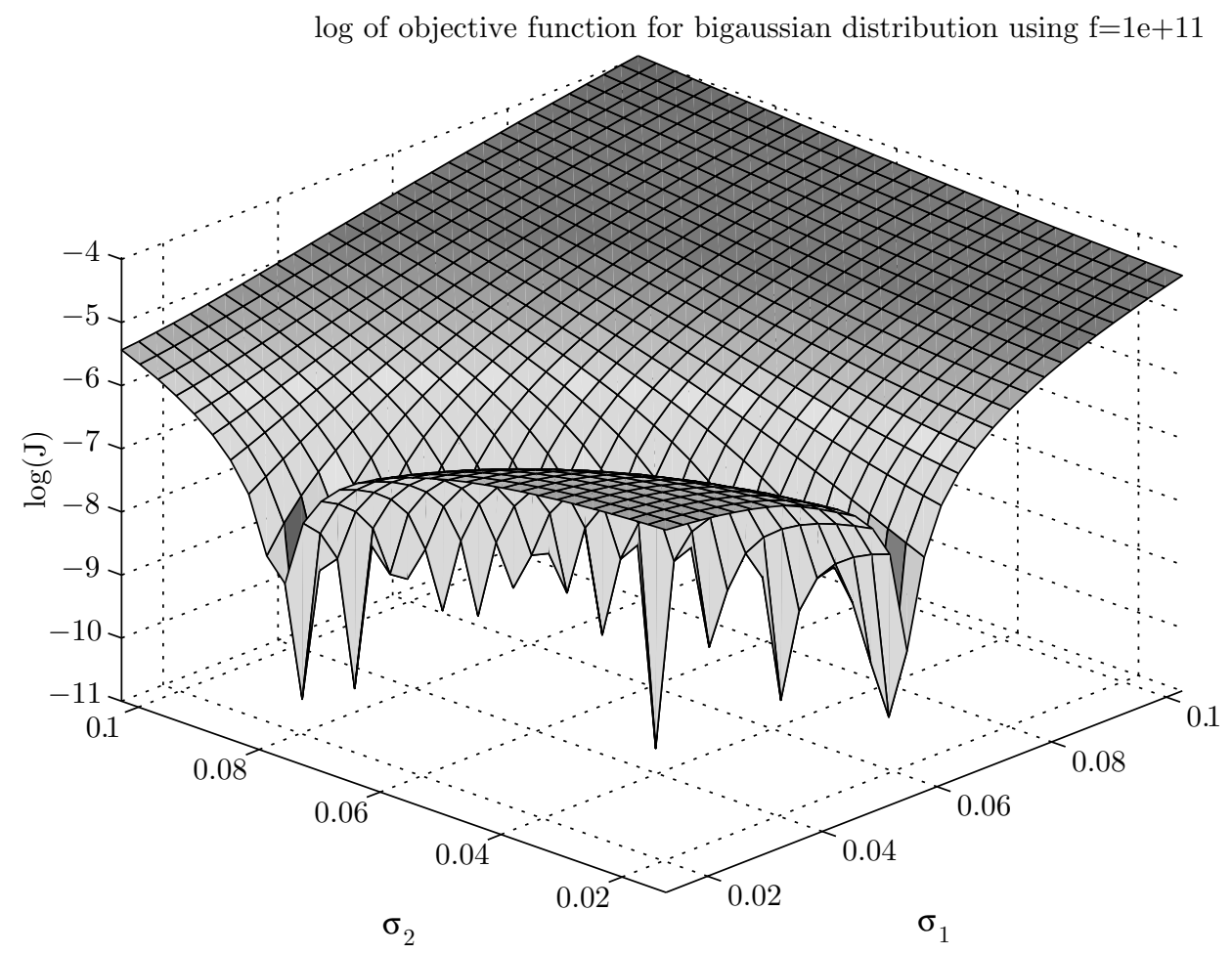

FIG. 13. The log of the objective function for the bi-Gaussian distribution inverse problem versus $\sigma_{1}$ and $\sigma_{2}$ using a frequency of $10^{11} \mathrm{~Hz}$.

For the discrete case involving two atoms, each value of $\tau$ was readily determined if the volume proportions were known. Likewise, the volume proportion could be determined given the values of each relaxation time. However, the problem of determining all threeparameters simultaneously is under-determined, but in certain situations, interrogating with a different frequency (in particular, greater than the critical frequency if the original interrogating frequency is smaller) can provide enough new information for the volume proportions and the relaxation times to be determined simultaneously.

The uniform distribution is generally straightforward to optimize; each endpoint of the distribution was determined, on average, to about three decimal places of accuracy.

The inverse problem involving the log-normal distribution, or rather a Gaussian distribution on the logarithms of the relaxation times, behaved similarly to the discrete distribution, and therefore the previous solution methods worked well. The mean of the distribution was determined to about four decimal places in log-space. The standard deviation was also determined quite accurately (about three decimal places), but it was evident in the surface plots that the objective function was particularly insensitive to the standard deviation. Any amount of noise in the system may prevent accurate results.

Finally, we considered a log-bi-Gaussian distribution of relaxation times. As in the Gaussian distribution problem, the objective function was insensitive to the standard 
deviations. Therefore, even with quite accurate estimates for the means, no usable estimates for the standard deviations could be found. However, in contrast, rather accurate solutions for both of the means could be determined using substantially incorrect estimates for the standard deviations. Still, the effect of the standard deviation on the objective function is sufficient to be able to distinguish distributions which are fairly broad (large standard deviations) from those that are nearly delta functions (very small standard deviations). This is important as it suggests that systems which truly have continuous distributions for their parameters should not be modelled using discrete distributions. Furthermore, an objective function of the type in this report may be used to create a measure of the error induced in the signal by opting to use a discrete distribution instead of a continuous one when it is sufficiently helpful for computational simplicity to do so.

In fact, homogenization techniques determine single values of relaxation times which produce signals that closely match those generated with more complex mechanisms (such as a distribution of Debye polarization mechanisms); for example, see [7]. It turns out that from our analysis here that for lower frequencies $\left(\omega_{L}<\omega_{c}=1 / \tilde{\tau}\right)$ the effective relaxation time is simply the weighted average of the actual relaxation times (or for continuous distributions, the integral of $\tau$ with respect to the distribution). However, for higher frequencies $\left(\omega_{H}>\omega_{c}=1 / \tilde{\tau}\right)$ the effective relaxation time is actually the inverse of the weighted average of inverses (or the inverse of the integral of $1 / \tau$ with respect to the distribution in the continuous case). For example,

$$
\tau_{e}=\frac{1}{\sum_{i} \frac{\alpha_{i}}{\tau_{i}}},
$$

where $\alpha_{i}$ represents the volume fraction of the material with discrete relaxation times $\tau_{i}$. Both the low frequency and high frequency estimates are common results in homogenization theory. However, any homogenization procedure which does not take the interrogating frequency into account will not be able to determine which is the correct effective relaxation time for the situation.

Lastly, it should be noted that the procedure of traversing a "line of best fit", as done in this report, is only a valid approach to solving nonlinear optimization problems (which have relations that are relatively optimal) if the equation of this curve is known. Other possible solution methods for more general problems include taking an "arbitrary orthogonal step", or resorting to a simplex search for those parts of the inverse problem that are particularly difficult for the gradient based methods.

10. Acknowledgements. This research was supported in part by the U.S. Air Force Office of Scientific Research under grants AF0SR F49620-01-1-0026, AFOSR FA9550-041-0220 and in part by the National Institute of Aerospace (NIA) and NASA under grant NIA/NCSU-03-01-2536-NC. The authors would like to thank Dr. Richard Albanese of the AFRL, Brooks AFB, and Dr. William P. Winfree, NASA Langley Research Center, for their valuable comments and suggestions during the course of this research. 


\section{REFERENCES}

[1] R.A. Albanese, H.T. Banks and J.K. Raye, Nondestructive evaluation of materials using pulsed microwave interrogating signals and acoustic wave induced reflections, Inverse Problems 18 (2002), 1935-1958. MR1955927 (2003k:78021)

[2] R.A. Albanese, R.L. Medina and J.W. Penn, Mathematics, medicine and microwaves, Inverse Problems 10 (1994), 995-1007. MR.1296358

[3] R.A. Albanese, J.W. Penn and R.L. Medina, Short-rise-time microwave pulse propagation through dispersive biological media, J. Optical Society of America A 6 (1989), 1441-1446.

[4] J.C. Anderson, Dielectrics, Chapman and Hall, London, 1967.

[5] H.T. Banks and K.L. Bihari, Modelling and estimating uncertainty in parameter estimation, Inverse Problems 17 (2001), 95-111. MR.1818494 (2002d:35215)

[6] H.T. Banks and V. A. Bokil, A computational and statistical framework for multidimensional domain acoustooptic material interrogation, Quarterly of Applied Mathematics 63 (2005), 156-200. MR 2126573(2005j:78032)

[7] H.T. Banks, V.A. Bokil, D. Cioranescu, N.L. Gibson, G. Griso and B. Miara, Homogenization of periodically varying coefficients in electromagnetic materials, CRSC-TR05-05, January, 2005; J. Scientific Computing, to appear.

[8] H.T. Banks, D. Bortz, G.A. Pinter and L.K. Potter, Modeling and imaging techniques with potential for application in bioterrorism, Chapter 6 in Bioterrorism: Mathematical Modeling Applications in Homeland Security, (H.T. Banks and C. Castillo-Chavez, eds.), Frontiers in Applied Mathematics, SIAM, Philadelphia, 2003, 129-154. MR2036539(2004k:92003)

[9] H.T. Banks and D.M. Bortz, Inverse problems for a class of measure dependent dynamical systems, J. Inverse and Ill-posed Problems, 13 (2005), 103-121. MR2147300 (2006a:34225)

[10] H.T. Banks, D.M. Bortz and S. E. Holte, Incorporation of variability into the modeling of viral delays in HIV infection dynamics, Math Biosci., 183 (2003), 63-91. MR.1965457 (2004b:92032)

[11] H.T. Banks, M.W. Buksas and T. Lin, Electromagnetic Material Interrogation Using Conductive Interfaces and Acoustic Wavefronts, Frontiers in Applied Mathematics, Vol. FR21, SIAM, Philadelphia, PA, 2000. MR1787981(2001k:78036)

[12] H. T. Banks and N. L. Gibson, Well-posedness in Maxwell systems with distributions of polarization relaxation parameters, Applied Mathematics Letters 18 (2005), 423-430. MR.2124300(2005i:35253)

[13] H. T. Banks and N. L. Gibson, Electromagnetic inverse problems involving distributions of dielectric mechanisms and parameters, CRSC-TR05-29, August, 2005.

[14] H.T. Banks, N.L. Gibson and W.P. Winfree, Gap detection with electromagnetic terahertz signals, Nonlinear Analysis: Real World Applications 6 (2005), 381-416. MR2111660 (2005m:78012)

[15] H.T. Banks and K. Kunisch, Estimation Techniques for Distributed Parameter Systems, Birkhäuser, Boston, 1989. MR1045629 (91b:93085)

[16] H.T. Banks, A.J. Kurdila and G. Webb, Identification of hysteretic control influence operators representing smart actuators, Part I: Formulation, Mathematical Problems in Engineering, 3 (1997), $287-328$.

[17] H.T. Banks, A.J. Kurdila and G. Webb, Identification of hysteretic control influence operators representing smart actuators: Part II, Convergent approximations, J. of Intelligent Material Systems and Structures, 8 (1997), 536-550.

[18] H.T. Banks, N.G. Medhin and G.A. Pinter, Multiscale considerations in modeling of nonlinear elastomers, CRSC-TR03-42, October, 2003; J. Comp. Meth. Sci. and Engr., to appear.

[19] H.T. Banks, N.G. Medhin and G.A. Pinter, Nonlinear reptation in molecular based hysteresis models for polymers, Quarterly Applied Math., 62 (2004), 767-779. MR2104273 (2005f:74022)

[20] H.T. Banks and G.A. Pinter, Maxwell systems with nonlinear polarization, Nonlinear Analysis: Real World Applications, 4 (2003), 483-501. MR.1956350 (2003k:78026)

[21] H.T. Banks and G.A. Pinter, High-frequency pulse propagation in nonlinear dielectric materials, Nonlinear Analysis: Real World Applications, 5 (2004), 597-612. MR2079271 (2005h:78019)

[22] H.T. Banks and G.A. Pinter, A probabilistic multiscale approach to hysteresis in shear wave propagation in biotissue, SIAM J. Multiscale Modeling and Simulation 3 (2005), 395-412. MR2122994 (2005k:74058)

[23] J.G. Blaschak and J. Franzen, Precursor propagation in dispersive media from short-rise-time pulses at oblique incidence, J. Optical Society of America A 12 (1995), 1501-1512. 
[24] C. J. F. Böttcher and P. Bordewijk, Theory of Electric Polarization, Vol. II, Elsevier, New York, 1978.

[25] R.W. Boyd, Nonlinear Optics, Academic Press, Boston, 1992.

[26] M. Caputo, Rigorous time domain responses of polarizable media, Annali di Geofisica XL (1997), 423-434.

[27] W.T. Coffey, Y.P. Kalmykov and S.V. Titov, Inertial effects in anomalous dielectric relaxation, $J$. Molecular Liquids 114 (2004), 35-41.

[28] K.S. Cole and R.H. Cole, Dispersion and absorption in dielectrics, J. Chemical Phys. 9 (1941), 341-351.

[29] M. Davidian and D.M. Giltinan, Nonlinear Models for Repeated Measurement Data, Monographs on Statistics and Applied Probability, Vol. 62, Chapman and Hall, New York, 1995.

[30] P. Debye, Polar Molecules, Chemical Catalogue Co., New York, 1929.

[31] R.S. Elliott, Electromagnetics: History, Theory and Applications, IEEE Press, New York, 1993.

[32] K.R. Foster and H.P. Schwan, Dielectric properties of tissues and biological materials: A critical review, Critical Rev. in Biomed. Engr. 17 (1989), 25-104.

[33] C. Gabriel, Compilation of the dielectric properties of body tissues at RF and microwave frequencies, Technical Report AL/OE-TR-1996-0037, USAF Armstrong Laboratory, Brooks AFB, TX, 1996

[34] C. Gabriel, S. Gabriel and E. Corthout, The dielectric properties of biological tissues: I. Literature survey, Phys. Med. Biol. 41 (1996), 2231-2249.

[35] S. Gabriel, R.W. Lau and C. Gabriel, The dielectric properties of biological tissues: II. Measurements in the frequency range $10 \mathrm{~Hz}$ to $20 \mathrm{GHz}$, Phys. Med. Biol. 41 (1996), 2251-2269.

[36] S. Gabriel, R.W. Lau and C. Gabriel, The dielectric properties of biological tissues: III. Parametric models for the dielectric spectrum of tissues, Phys. Med. Biol. 41 (1996), 2271-2293.

[37] O.P. Gandhi, B.Q. Gao and J.Y. Chen, A frequency-dependent finite-difference time-domain formulation for general dispersive media, IEEE Trans. Microwave Theory and Tech., 41 (1993), 658-665.

[38] N. L. Gibson, Terahertz-Based Electromagnetic Interrogation Techniques for Damage Detection, Ph.D. Thesis, N. C. State University, Raleigh, 2004.

[39] W.D. Hurt, Multiterm Debye dispersion relations for permittivity of muscle, IEEE Trans. Biomed. Engr. 32 (1985), 60-64.

[40] J.D. Jackson, Classical Electrodynamics, John Wiley and Sons, New York, 1975. MR0436782 $(55: 9721)$

[41] R.M. Joseph and A. Taflove, FDTD Maxwell's equations models for nonlinear electrodynamics and optics, IEEE Trans. Antennas and Propag., 45 (1997), 364-374.

[42] M.A. Krasnosel'skii and A.V. Pokrovskii, Systems with Hysteresis, Nauka, Moscow, 1983; translated from the Russian, Springer-Verlag, Berlin, 1989. MR.0742931(86e:93005)

[43] I.D. Mayergoyz, Mathematical Models of Hysteresis, Springer-Verlag, New York, 1991. MR1083150 (92b:78001)

[44] E.J. McShane, Generalized curves, Duke Math J. 6 (1940), 513-536. MR0002469 (2:59d)

[45] E.J. McShane, Relaxed controls and variational problems, SIAM J. Control 5 (1967), 438-485. MR0218949 (36:2033)

[46] N. Miura, S. Yahihara, and S. Mashimo, Microwave dielectric properties of solid and liquid foods investigated by time-domain reflectometry, J. Food Science 68 (2003), 1396-1403.

[47] P.G. Petropoulos, On the time-domain response of Cole-Cole dielectrics, IEEE Trans. Antennas and Propag., (2005), to appear.

[48] O. Pironneau, Optimal Shape Design for Elliptic Systems, Springer-Verlag, New York, 1984. MR0725856 (86e:49003)

[49] W.W. Schmaedeke, Optimal control theory for nonlinear vector differential equations containing measures, J. SIAM Ser. A Control 3 (1965), 231-280. MR.0189870 (32:7288)

[50] M.H. Schultz, Spline Analysis, Prentice-Hall, Englewood Cliffs, NJ, 1973. MR0362832 (50:15270)

[51] L. Schumaker, Spline Functions: Basic Theory, J. Wiley \& Sons, New York, 1981. MR0606200 (82j:41001)

[52] E.R Von Schweidler, Studien über anomalien im verhalten der dielektrika, Ann. Physik 24 (1907), $711-770$.

[53] K.W. Wagner, Zur theorie der unvollkommenen dielektrika, Ann. Physik 40 (1913), 817-855.

[54] J. Warga, Relaxed variational problems, J. Math. Anal. Appl. 4 (1962), 111-128. MR0142020 (25:5415a)

[55] J. Warga, Functions of relaxed controls, SIAM J. Control 5 (1967), 628-641. MR 0226474(37:2064a) 
[56] J. Warga, Optimal Control of Differential and Functional Equations, Academic Press, New York, 1972. MR0372708 (51:8915)

[57] M.L. Williams and J.D. Ferry, Second approximation calculations of mechanical and electrical relaxation and retardation distributions, J. Poly. Sci. 11 (1953), 169-175.

[58] L.C. Young, Generalized curves and the existence of an attained absolute minimum in the calculus of variations, C. R. Soc. Sci. et Lettres, Varsovie, Cl. III, 30 (1937), 212-234.

[59] L.C. Young, Necessary conditions in the calculus of variations, Acta Math. 69 (1938), 239-258. 